\title{
Hormetic response and transcriptome profiling reveal the mechanism underlying metabolic and cuticular resistance to host-specific terpenoid defences in an emerging insect pest, Pagiophloeus tsushimanus (Coleoptera: Curculionidae)
}

Shouyin Li

Nanjing Forestry University

Hui Li

Nanjing Forestry University

Jingting Wang

Nanjing Forestry University

Cong Chen

Nanjing Forestry University

Dejun Hao ( $\boldsymbol{\sigma}$ djhao@njfu.edu.cn )

Nanjing Forestry University https://orcid.org/0000-0001-7743-9968

\section{Research Article}

Keywords: Feeding bioassays, Hormetic response, RNA-seq, CYP450-mediated metabolic resistance, CPmediated cuticular resistance, Insect-plant coevolutionary adaptation

Posted Date: August 23rd, 2021

DOI: https://doi.org/10.21203/rs.3.rs-831863/v1

License: (c) (i) This work is licensed under a Creative Commons Attribution 4.0 International License.

Read Full License 


\section{Abstract}

The resistance mechanisms evolved by insects to overcome host-plant allelochemicals are a key consideration in pest management. Camphor oil (EO) and its main component (i.e., D-camphor) form a specific terpenoid-defensive system in camphor trees, Cinnamomum camphora. However, an emerging insect pest, Pagiophloeus tsushimanus, has recently caused serious damage to this intractable plant species and is largely elusive. Here, we used feeding bioassays and RNA-seq to investigate the mechanism underlying the resistance of the beetle to host-specific terpenoid defences. First, a hormetic response in both larval weight and developmental time, which is a highly generalized dose-response phenomenon in toxicology but occurs infrequently in the context of insect-plant interactions, was observed in terpenoid-feeding individuals. Then, comparative transcriptome analysis between terpenoidfeeding and control groups indicated that both CYP450-mediated metabolic resistance and CP-mediated cuticular resistance were jointly employed to cope with terpenoid-induced stress. In addition, a small portion of genes involved in the glucose transport pathway were upregulated at the low D-camphor dose, suggesting that an extra intake of glucose used for larval growth may contribute to a hormetic response. These findings suggested that the dual terpenoid resistance mechanisms in this specialist are an essential precondition for the hormetic response in larval growth, ultimately contributing to the widespread successful colonization of host camphor trees. Overall, our study will open new avenues for understanding insect-plant coevolutionary adaptation and developing durable pest control strategies.

\section{Introduction}

Plants and herbivores are continuously engaged in a struggle for survival and employ different strategies to prevent the evolution of resistance or adaptation to defensive compounds (Stahl et al., 2018). The preexisting constitutive defences in plants (e.g., toxic secondary metabolites and proteins) are everincreasingly investigated for the development of novel biopesticides (Mossa, 2016; Ntalli et al., 2019; Magalhaes et al., 2020; Maurya et al., 2020). Efficient plant resistance exerts strong selection pressure on insects, which in turn develop ways to cope with the deleterious effect of phytotoxin in a stepwise process (Heckel, 2014; Heidel-Fischer and Vogel, 2015; Petschenka and Agrawal, 2016). In recent decades, most reports have documented a suite of factors that confer insect resistance to xenobiotics, including metabolic resistance, cuticular resistance (i.e., penetration resistance), target-site resistance, and behaviour resistance (Tangtrakulwanich and Reddy, 2014). There is, however, a lack of understanding of how these different mechanisms interact (additively, synergistically, or even antagonistically) and to what extent they affect the resistance phenotype.

Evidence is accumulating that cytochrome P450 monooxygenases (CYP450s) are pivotal to metabolic resistance (Hrycay and Bandiera, 2015; Lu et al., 2021; Nauen et al., 2021), and very recently, a comprehensive review discussing the roles of CYP450s in response to phytochemicals was published elsewhere (Vandenhole et al., 2021). Notably, despite considerable progress in the identification of shortterm inducible P450s, the relationship between upregulated expression and metabolism remains underexplored, and the extent to which this response is biologically functional should be further 
determined. Moreover, few studies have examined whether genes induced by initial exposure become constitutively overexpressed after long-term adaptation (Wybouw et al., 2015; Müller et al., 2017; Salehipourshirazi et al., 2020). CYP450-mediated detoxification in insect adaptation to xenobiotics has been studied extensively, while other mechanisms exist outside of this paradigm. Cuticular resistance refers to modifications in the cuticle (i.e., thickening of the cuticle or altered cuticle composition) that ultimately minimize penetration of xenobiotics into insect bodies (Balabanidou et al., 2018). It is commonly believed that structural cuticular proteins (CPs), as basic components of the cuticle, are frequently overexpressed to accomplish cuticular thickening. To date, a small number of CP genes associated with cuticular resistance to insecticides have been identified, but a few studies have proven that CPs also confer resistance to host allelochemicals (Awolola et al., 2009; Puinean et al., 2010; Nkya et al., 2014; Fang et al., 2015; Yahouedo et al., 2017a).

To reveal the mechanism underlying metabolic and cuticular resistance, next-generation RNA sequencing (RNA-seq) has always been a powerful tool for characterizing transcriptomic responses in the complex context of gene expression and regulation (Todd et al., 2016). Extensive RNA-seq analysis has demonstrated that one or more CYP450 genes are inevitably activated by quercetin (Zhang et al., 2018), nicotine (Ramsey et al., 2014; Zhang et al., 2018), gossypol (Celorio-Mancera et al., 2011), xanthotoxin (Cheng et al., 2017), tomatine (Li et al., 2019), and several terpenoids (e.g., plant essential oil) (Liao et al., 2016; Huang et al. 2018a). Additionally, a common finding in certain herbivore transcriptome studies has shown enrichment of the 'structural constituent of the cuticle', which is closely related to CPs (Fescemyer et al., 2013; Kliot et al., 2014; Roy et al., 2016; Schweizer et al., 2017). These studies have provided a battery of candidate genes (e.g., the CYP6 family and a suite of CPs) potentially involved in adaptation to plant defensive chemicals. Nevertheless, only a handful of models for insect-plant interactions offered unbiased transcriptional profiles related to host plant adaptations in herbivorous insects, and few studies have revealed these two resistance mechanisms simultaneously in the same experimental model.

The insect pest Pagiophloeus tsushimanus (Morimoto) is a trunk-boring specialist herbivore with only camphor trees as its preferred host in natural environments (Chen et al., 2020; Li et al., 2021). Since this weevil was reported in 2014, it has rapidly reached epidemic levels in Shanghai, China. The camphor tree Cinnamomum camphora (L.) Presl (Laurales: Lauraceae), has been widely exploited as a shade tree or as industrial material in several countries, and it is less likely to be vulnerable to various herbivorous insects because of its specific terpenoid defences (e.g. a class of monoterpene-derived metabolites such as Dcamphor) (Li et al., 2021). Thus, it would be interesting to explore how this pest could mount an efficient counter-defence response. In this work, we first evaluated larval performance with different dosages of terpenoid-infused artificial diets (i.e., treatment with camphor oil and D-camphor in the diet) by measuring a set of developmental parameters (i.e., lethal concentration, larval viability, body weight and developmental time). To determine whether $P$. tsushimanus exhibits both metabolic and cuticular resistance to host-specific terpenoid metabolites, the transcriptional responses of $P$. tsushimanus larvae to different dosages of camphor oil and D-camphor were investigated by using RNA-seq analysis. Our findings not only provide a new example of the molecular mechanisms underlying insect adaptation to plant secondary metabolites but also establish a foundation for future pest management. 


\section{Materials And Methods \\ Plant materials, insects and reagents}

The camphor tree Cinnamomum camphora (L.) Presl, used as an artificial diet substrate, was provided by Nanjing Forestry University $\left(32^{\circ} 4^{\prime} 43^{\prime \prime} \mathrm{N} / 118^{\circ} 48^{\prime} 30^{\prime \prime} \mathrm{E}\right)$, Jiangsu Province, China, and never treated with pesticides. Twigs aged 1-3 years were collected and crushed and then passed through a 100 mesh screen. The resulting powder was stored at $4{ }^{\circ} \mathrm{C}$ for subsequent experiments.

Pagiophloeus tsushimanus was obtained from a culture established at the Entomology Biological Laboratory, Nanjing Forestry University (EBL, NJFU), Jiangsu Province, China, in 2019 and originally collected from C. camphora plantations in Songjiang District, Shanghai Province, China $\left(30^{\circ} 56^{\prime} 6.15^{\prime \prime} \mathrm{N}\right.$, $\left.121^{\circ} 12^{\prime} 32.76^{\prime \prime} \mathrm{E}\right)$. The breeding system applied to $P$. tsushimanus eggs, larvae, pupae and adults was described in our previous study (Li et al., 2021). In brief, eggs were deposited in a Petri dish $(5 \mathrm{~cm}$ i.d. $\times 1$ $\mathrm{cm}$ ) with a water-soaked cotton pad for incubation, and neonate larvae were then individually reared in a six-well Petri dish $(3.5 \mathrm{~cm}$ i.d. $\times 1.5 \mathrm{~cm}$ each well) with artificial diets. The formulation of the artificial diets was $50 \%(\mathrm{w} / \mathrm{w})$ distilled water, $40 \%(\mathrm{w} / \mathrm{w})$ host-plant powder, $5 \%(\mathrm{w} / \mathrm{w})$ agar, $3 \%(\mathrm{w} / \mathrm{w})$ sucrose, $1 \%$ $(\mathrm{w} / \mathrm{w})$ yeast, $0.5 \%(\mathrm{w} / \mathrm{w})$ sorbic acid and $0.5 \%(\mathrm{w} / \mathrm{w})$ sodium benzoate. Both eggs and larvae were kept in an environmental incubator (MTI-201B, Tokyo Rikakikai, Japan) at $28 \pm 1^{\circ} \mathrm{C}$ with a $70 \pm 5 \%$ relative humidity and a photoperiod of 16:8 h (L:D).

Essential oil (EO) (99\% purity, S27261) derived from the branches of the camphor tree and D-camphor (96\% purity, C106734, CAS: 464-49-3) were purchased from Sigma-Aldrich (St. Louis, MO, USA).

\section{Feeding bioassays: larval performance with terpenoid- infused artificial diets}

To evaluate their dose response to host terpenoids, neonate larvae were fed artificial diets mixed with different doses of EO $\left(0,2,4,6,10,12,30,50,100,150\right.$, and $\left.200 \mathrm{mg} \mathrm{g}^{-1}(\mathrm{w} / \mathrm{w})\right)$ and D-camphor $(0,5,10$, $15,17,20,30,50,70,100,120,150,170$, and $\left.200 \mathrm{mg} \mathrm{g}^{-1}(\mathrm{w} / \mathrm{w})\right)$. Since pure D-camphor is solid at room temperature, it was added directly to the host-plant powder and dissolved through vortexing to prepare a homogenous mixture for formulating the diets. EO was pre-dissolved as a liquid in a hydrosolvent. No fewer than sixty individuals were used for each dose treatment. The mortality at $72 \mathrm{~h}$ was recorded, and follow-up observations were performed $24 \mathrm{~h}$ later to confirm mortality. Data generated from this trial were used to determine the chronic working doses of $\mathrm{EO}$ and $\mathrm{D}$-camphor in accordance with the maximum nonlethal concentration $\left(\mathrm{LC}_{00}\right)$ and lethal concentration killing $15 \%$ of the beetle population $\left(\mathrm{LC}_{15}\right)$.

According to the results of the dose-response assessment, a total of 800 neonate larvae were continuously reared on artificial diets containing two doses of $\mathrm{EO}$ and $\mathrm{D}$-camphor $\left(\mathrm{LC}_{00}\right.$ and $\left.\mathrm{LC}_{15}\right)$, respectively, until pupation (200 larvae per treatment). The control group of larvae (200 larvae) were fed a terpenoid-free diet. These larvae were segregated and reared on fresh diets, which were replaced every 
three days, and their weight, cumulative mortality and developmental time were simultaneously recorded. The duration of the pupal stage and pupation rates were also determined.

\section{RNA sample collection, library construction, and Illumina sequencing}

The treatment procedures were carried out as described above. Several vigorous larvae were randomly sampled for RNA sequencing (RNA-seq) after continuous exposure for 36 days (approximately 4th-instar larvae). Three replicates with four larvae per replicate were used for each treatment (total of 12 larvae per treatment). All RNA-seq samples were processed for total RNA extraction using TRIzol Reagent (TaKaRa, Japan) according to the manufacturer's protocol. RNA quantity was checked using a Nanodrop 2000 (Thermo Scientific, Waltham, MA, United States). RNA integrity was monitored on $1 \%$ agarose gels. The complementary DNA (cDNA) libraries were generated with NEBNext Ultra ${ }^{\mathrm{TM}}$ RNA Library Prep Kits for Illumina (NEB, Beverly, MA, United States) following the manufacturer's protocol. The high quality of the libraries was confirmed using a high-sensitivity DNA chip on an Agilent Bioanalyzer 2100 (Agilent Technologies, Palo Alto, CA, United States). Paired-end sequencing was conducted on the Illumina HiSeq 2000 platform by Shanghai Majorbio Bio-pharm Biotechnology Co., Ltd. (Shanghai, China).

\section{Transcriptome assembly and functional annotation}

Raw reads were filtered by removing reads containing adapters and $>10 \%$ ambiguous ' $\mathrm{N}$ ' nucleotides and low-quality reads (defined as reads with $>20 \%$ of the bases having quality scores $<10$ ) in SeqPrep software (https://github.com/jstjohn/SeqPrep) before transcriptome assembly. The Q20, Q30 and GC content of the cleaned datasets were calculated to assess sequencing quality in fastx_toolkit_0.0.14 software (http://hannonlab.cshl.edu/fastx_toolkit/). These clean reads of all samples were pooled and then de novo assembled without a reference genome in the TRINITY program with default parameters (Grabherr et al., 2011). The nonredundant sequences were obtained by removing sequences showing redundancy in CD-HIT software (http://weizhongli-lab.org/cd-hit/), and the longest transcript of each gene was defined as a 'unigene'. To annotate the obtained unigenes, six public databases, namely, the NCBI nonredundant proteins (NR, e-value $\left.\leq 1.0 \times 10^{-5}\right)$, Swiss-Prot (e-value $\leq 1.0 \times 10^{-5}$ ), Protein families (Pfam, e-value $\left.\leq 1.0 \times 10^{-5}\right)$, Clusters of Orthologous Groups (COG, e-value $\left.\leq 1.0 \times 10^{-5}\right)$, (Gene Ontology $\left(G O\right.$, e-value $\left.\leq 1.0 \times 10^{-5}\right)$, and Kyoto Encyclopedia of Genes and Genomes (KEGG, e-value $\leq 1.0 \times 10^{-10}$ ) databases, were searched.

\section{Gene expression analysis and $\mathrm{GO}$ enrichment analysis}

To quantify the gene expression level, sequenced reads of each sample were mapped to the assembly contigs using RSEM software with default parameters (Li and Dewey, 2011). The mapped read counts (Fragments Per Kilobase of exon model per Million mapped fragments, FPKM) were calculated by Cuffdiff version 2.1.0 (http://cole-trapnell-lab.github.io/cufflinks/). The DESeq2 program provided statistical routines for determining differentially expressed genes (DEGs) between the control group (CK) and terpenoid-exposed groups (i.e., EO and D-camphor treatments) (Anders and Huber, 2010). Fold 
change (FC) values for gene expression were considered significant if $P$-value $<0.05$ (false discovery rate $\leq 0.01)$ and $|\log 2 \mathrm{FC}| \geq 1$. GO enrichment analysis was performed for the identified DEGs of interest using the Goatools program (https://github.com/tanghaibao/GOatools), and an adjusted $P$-value $<0.05$ was the threshold value for significant enrichment results.

\section{Weighted gene co-expression network analysis (WGCNA)}

Using the WGCNA package in $\mathrm{R}$

(https://horvath.genetics.ucla.edu/html/CoexpressionNetwork/Rpackages/WGCNA/), a Pearson's correlation matrix was constructed for all pairs of genes, and a weighted adjacency matrix was constructed with a power function: (where $a_{m n}$ is the adjacency between gene $m$ and gene $n, S_{m n}$ is Pearson's correlation between gene $m$ and gene $n$, and $\beta$ is the soft thresholding parameter allowing for a maximal correlation coefficient). The power of $\beta=4$ (model fitting index $R^{2}=0.89$ ) was set in accordance with the scale-free topology criterion (Supplementary Fig. S2). The adjacency matrix was further transformed into a topological overlap matrix using the WGCNA package to determine the network connectivity of the genes. The genes with similar expression profiles were classified into gene modules by using the cut-tree Hybrid function in Dynamic Tree Cut package version 1.62 with default parameters ( minModuleSize $=30$ and mergeCutHeight $=0.25$ ). To identify the modules that were significantly associated with terpenoid exposure treatments, module significance (MS) was set as Spearman's correlation coefficient, and plotted in the WGCNA package. A module with a high MS value was considered to be a key module. Hub genes were defined based on the degree of network connectivity using the WGCNA package (Langfelder and Horvath, 2008). The top 30 target genes (i.e., those with the highest connectivity) were selected as hub genes associated with terpenoid exposure treatments and visualized using Cytoscape version 3.4 (Shannon et al., 2003).

\section{Real-time quantitative PCR (RT-qPCR) validation}

To validate the RNA-seq results, 14 genes of interest were selected for RT-qPCR experiments. Insect samples were prepared as described above for RNA-Seq library preparation. Three biological replicates were included for each treatment, and each biological replicate contained three technical replicates. The cDNA of each sample was synthesized using a HiScript 1st Strand cDNA Synthesis Kit according to the manufacturer's protocol (Vazyme Biotech Co., Ltd, Nanjing, China). RT-qPCR experiments were performed on an Applied Biosystem 7500 Real-Time PCR System (Thermo Fisher, Massachusetts, United States) using SYBR Premix Ex Taq II (TaKaRa, Japan) according to the manufacturer's protocol. The reaction conditions were as follows: 5 min at $95^{\circ} \mathrm{C}$, followed by 40 cycles of $10 \mathrm{~s}$ at $95^{\circ} \mathrm{C}$ and $40 \mathrm{~s}$ at $60{ }^{\circ} \mathrm{C}$. TATA-binding protein (TBP) was selected as the housekeeping gene based on the geNorm calculation results. Specific primers for each gene were designed using Primer Premier version 5.0 (Supplementary Table S5) (Singh et al., 1998) and synthesized at Bgi Gene Technology Co., Ltd. (Beijing, China). Relative expression levels were determined by the $2^{-\triangle \Delta C t}$ method (Schmittgen and Livak, 2008).

\section{Data analyses}


The mortality rates of the neonate larvae reared on terpenoid-infused diets after $72 \mathrm{~h}$ were regressed against the concentrations using a log-logistic regression model, and the lethal concentrations (i.e., $\mathrm{LC}_{15}$ and $\mathrm{LC}_{50}$ ) was calculated using the fitted function: See function in the supplementary files section, where $y$ and $x$ are response and dose variables, respectively. This model is generally applied to describe logistic dose responses in pharmacology and chemistry. To combine the effects of terpenoid exposure on developmental time (DT), body weight (BW), and larval viability (LV) in feeding bioassays, we calculated a relative performance index (RPI) for each developmental stage using (Soto et al., 2014). Furthermore, we used the chi-square contingency table test $(P<0.05)$ to determine the maximum nonlethal concentration $\left(\mathrm{LC}_{00}\right)$ in the feeding bioassays at low concentrations, and Yates's correction for continuity was performed when expected frequencies were below five. The developmental durations and larval and pupal weights at different stages were analysed using the Kruskal-Wallis ANOVA test $(P<0.05)$, and a post hoc test was conducted to evaluate the differences among the treatments. The age-stage specific survival rates of the larvae reared on terpenoid-infused artificial diets were compared using Kaplan-Meier survival analysis with the log-rank test. Statistical significance of RT-qPCR data was determined using one-way ANOVA followed by Tukey's HSD test $(P<0.05)$. To verify the reliability of RNA-Seq data in gene expression analysis, Pearson's correlation between fold changes (Log2 transformed) in RT-qPCR and RNA-Seq results was analysed.

All analyses were conducted in SPSS version 20.0 software (IBM SPSS Statistics, Chicago, IL, United States), and plots were generated with OriginPro version 9.0 software (OriginLab Inc., Northampton, United Kingdom).

\section{Results}

\section{Effect of EO and D-camphor on larval performance}

When neonate larvae were reared on an artificial diet with different low concentrations of EO and Dcamphor for $3 \mathrm{~d}$, significant deaths initially occurred at $12 \mathrm{mg} \cdot \mathrm{g}^{-1}$ and $20 \mathrm{mg} \cdot \mathrm{g}^{-1}$, respectively $(P<0.05$, Fig. 1a). Therefore, the $\mathrm{LC}_{00}$ values of the $E O$ and $\mathrm{D}$-camphor treatments were $10 \mathrm{mg} \cdot \mathrm{g}^{-1}$ and $17 \mathrm{mg} \cdot \mathrm{g}^{-1}$, respectively. As shown in Fig. 1b, the unique working concentrations of EO and D-camphor based on their calculated $\mathrm{LC}_{15}$ values used in subsequent bioassays were $30 \mathrm{mg} \cdot \mathrm{g}^{-1}$ and $41 \mathrm{mg} \cdot \mathrm{g}^{-1}$, respectively.

There were variations in developmental time, body weight, and larval viability between the terpenoidexposed and control groups (Fig. 2). The developmental time of larvae feeding on the terpenoid-infused diets was longer than that of the control group when they reached the 2 nd instar. However, a significant increase in the growth rate of larvae exposed to terpenoids at $\mathrm{LC}_{00}$ (EO_10mg_g \& D_camphor_17mg_g) was observed afterwards (Fig. 2a). Body weight was either unaffected or slightly inhibited at $\mathrm{LC}_{15}$ (EO_30mg_g \& D_camphor_41 mg_g), and we even found that the highest weight gain was observed at $\mathrm{LC}_{00}$, with a gain $10 \%$ greater than when terpenoid was absent in terms of pupal weight (Fig. 2b). Terpenoid exposure significantly affected the age-specific survival rate (log-rank test, chi-square = 
$247.772, P<0.001)$. The survivorship curves showed that the larvae reared on terpenoid-infused diets had rapidly declining survivorship from 9 to 24 days after egg hatching, and increasing doses of EO and Dcamphor markedly affected larval viability (Fig. 2c). Our final step was to combine the developmental parameters described above into a single index (i.e., the RPI) that serves as a proxy of overall performance. According to the heatmap, the populations reared on terpenoid-infused diets at lower dosages had greater z-scores than the control group, whereas the lowest value occurred at higher dosages (Fig. 2d).

\section{Transcriptome responses to EO and D-camphor exposure}

RNA-seq of $P$. tsushimanus whole larvae fed terpenoid-infused or terpenoid-free diets yielded a total of $794,599,342$ high-quality, clean reads, with a $42.94 \%-43.62 \%$ GC content and over $96 \%$ and $91 \%$ Q20 and Q30 values, respectively (Table S1). De novo assembly of transcriptome data resulted in 34,486 unigenes and 44,513 transcripts. The total and average lengths of these unigenes were 41,545,312 bp and 1204.7 bp, respectively, with an N50 (length N for which 50\% of all bases in the assembly were located in a unigene of length $<\mathrm{N}$ ) of $2,245 \mathrm{bp}$ and a mapped fragment percentage of $59.75 \%$ (Table S2).

A total of 34,452 unigenes were annotated by comparing them to the COG, GO, KEGG, NR, Swiss-Prot or Pfam databases (Table S3). As shown in Figure S1, NR, GO and KEGG annotation provided comprehensive information on gene function. The species distribution of the top BLAST hits in the NR database showed that 6303 unigenes $(34.1 \%$ ) had the highest scoring blast matches to Dendroctonus ponderosae, followed by Sitopjilus oryzae (3434 unigenes, 18.6\%) (Fig. S1a). The observed G0 terms for each domain (biological process, cellular component, and molecular function) and KEGG pathways for each category (metabolism, human diseases, organismal systems, cellular processes, environmental information processing, and genetic information processing) are detailed in Figure S1b and c.

To screen the genes potentially involved in $P$. tsushimanus resistance to host-specific terpenoid defence (i.e., EO and D-camphor), differentially expressed genes (DEGs) were detected between the terpenoidexposed and nonexposed groups (CK_vs_EO_10mg_g; CK_vs_EO_30mg_g; CK_vs_D_camphor_17mg_g; CK_vs_D

_camphor_41mg_g), with the criteria $P$-value $<0.05$ and $|\log 2 \mathrm{FC}| \geq 1$. In total, 14 upregulated genes and 26 downregulated genes were observed in the EO_10mg_g treatment, whereas 104 upregulated genes and 99 downregulated genes were observed in the EO_30mg_g treatment (Fig. 3a). Additionally, 116 upregulated genes and 48 downregulated genes were observed in the D_camphor_17mg_g treatment, whereas 143 upregulated genes and 68 downregulated genes were observed in the D_camphor_41mg_g treatment (Fig. 3b). The Venn diagram showed that 3, 111, 120, and 50 specific DEGs (i.e., DEGs occurring in only a particular treatment) were found in the EO_10mg_g, EO_30mg_g, D_camphor_17mg_g, and D_camphor_41 mg_g treatments, respectively (Fig. 4c). In addition, 18 common DEGs (i.e., DEGs occurring in all treatments) were significantly different between the terpenoid-exposed and nonexposed groups (Fig. 3c). 
To annotate the putative functions of the DEGs identified in each treatment, GO enrichment analysis was performed (Fig. 4). In brief, terpenoid exposure induced similar molecular responses in all groups: most upregulated unigenes were significantly enriched in monooxygenase activity related to CYP450s and structural constituent of cuticle related to CPs, while downregulated unigenes significantly enriched in protein folding and de novo posttranslational protein folding were only observed in the EO_10mg_g treatment (Fig. 4a). Subsequently, we placed emphasis on two GO pathways: monooxygenase activity (GO: 0004497) and structural constituent of cuticle (GO: 0042302). CYP450 genes accounted for $87.6 \%$ of the monooxygenase activity (Fig. 5a). Among these genes, several CYP450 genes were significantly upregulated after terpenoid exposure, notably the genes called 'TRINITY_DN986_C0_g1', 'TRINITY_DN32261_C0_g1', and 'TRINITY_DN1 2993_C0_g1' (Fig. 5b-e). Analogous to the results for the monooxygenase activity pathway, among the genes associated with structural constituent of cuticle pathway, $88.9 \%$ belonged to the CP gene family (Fig. 6a). We also found that most of the CP genes were commonly upregulated in all treatments, while a few genes were found to be downregulated after EO treatments, including the genes called 'TRINITY_DN3873_C0_g1' and 'TRINITY_DN17856_C0_g1' (Fig. 6be).

WGCNA was used to further identify the target genes involved in countering host-specific terpenoid defence. As shown in the gene dendrogram, the genes with similar expression patterns were globally divided into 13 unique module eigengenes using average linkage clustering (Supplementary Fig. S3). The relevance between each module and terpenoid-exposure treatment showed that the MEblack module had a higher correlation with D-camphor treatment than other modules (MS-value $=0.441$ ), whereas the MEtan module was the most relevant to EO treatment (MS-value $=0.787$ ) (Fig. 7a). Next, the top 30 genes with the highest connectivity (i.e., hub genes) in the MEblack and MEtan modules were used to analyse gene expression and co-expression networks (Fig. 7b-g). We found that the hub genes in the MEtan module exhibited higher expression levels in EO treatments than in D-camphor treatments (Fig. 7b \& c). With respect to hub genes in the MEblack module, there was no obvious difference in expression level between the EO and D-camphor groups (Fig. 7e \& f). Using co-expression networks, five core hub genes in the MEtan and MEblack modules were identified (Fig. 7d \& g). Remarkably, the core hub genes in the MEtan module, namely TRINITY_DN986_C0_g1, TRINITY_DN32261_C0_g1, TRINITY_DN12993_C0_g1, and TRINITY_DN1904_

C0_g1, belong to the CYP450 family, whereas the gene TRINITY_DN1820_C0_g1 is of unknow function (Fig. 7d). In the MEblack modules, the core hub genes, namely, TRINITY_DN39845_C0_g1, TRINITY_DN1984_C0_g1, TRINITY_DN624_C0_g1, and TRINITY_DN5193_C0_g1, were annotated to the cuticular protein family, whereas the gene TRINITY_DN129_C0_g1 was uncharacterized (Fig. 7g).

\section{Validation of common DEGs by RT-qPCR}

We inspected the expression of fourteen interesting unigenes encoding CYP450s and cuticular proteins across all terpenoid treatments by qRT-PCR (Supplementary Table S4). All the unigenes encoding cytochrome P450s were upregulated to varying degrees, which was highly consistent with the RNA-seq results (Pearson's $r=0.951, P<0.001$ ) (Fig. 8). Similar trends of upregulation of the unigenes encoding 
cuticular proteins between RT-qPCR and RNA-seq data were also observed (Pearson's $r=0.604, P<0.001$ ) (Fig. 9). These results supported the accuracy and reliability of the RNA-Seq data.

\section{Discussion}

\section{A hormetic response in larval growth}

Dose responses, known as the backbone of toxicology, are crucial to understanding the capacity of organisms (e.g., insect pests) to tolerate toxic exposures to chemical, biological, and physical agents (e.g., insecticides or phytotoxins) (Agathokleous and Calabrese, 2019). In this context, hormesis, a term that was coined by Southam and Ehrlich (1943), describes biphasic dose-response relationships separated into two dose zones, one (i.e., a relatively low dose) with stimulatory responses, and the other one (i.e., a relatively high dose) with detrimental effects. The results herein showed that hormesis seems to be a notable feature of the biological response of $P$. tsushimanus to host-specific terpenoid defences, as demonstrated by the rapid accumulation of body weight within a shorter developmental time when the individuals were exposed to a terpenoid-infused diet at $\mathrm{LC}_{00}$ (i.e., EO_10mg_g \& D_camphor_17mg_g). This phenomenon was also exemplified by studies on the response of cotton pests to gossypol, whereby a similar stimulatory effect was triggered by low gossypol concentrations (Stipanovic et al., 2006; Stipanovic et al., 2008; Celorio-Mancera et al., 2011). At present, there are two possible explanations for hormesis: 'overcompensation' theory and the principle of energy allocation. The former argues that hormesis is the result of both transient and sustained homeostasis overcompensation in which control systems with feedback responses can regulate growth to counteract the detrimental effects of toxic agents (Stebbing, 1998; Calabreseci, 1999), while the latter posits that the stress-exposed individuals shift the balance between energy-conflicting physiological trade-offs (e.g., reproduction vs. Iongevity), favouring one at the expense of another (Forbes, 2000; Jager et al., 2013). Although in this regard we have no data on the relevant parameters of adult reproduction and longevity to further support these hypotheses, the combination of these hypotheses suggests an evolutionary expectation that some emerging specialists that efficiently utilize host-plant chemicals as a feeding stimulus could build up a large population with damaging numbers in a short period, ultimately leading to an outbreak of insect pests.

In addition, the concept of phytotoxin-induced hormesis has not received sufficient attention, because its molecular basis either is completely unpredictable, or falls into a general pattern of activating stressresponse mechanisms that are generally beneficial to the organism (e.g., hormones, heat shock proteins and antioxidant enzymes) (Vaiserman, 2010; Yu et al., 2010). In evaluating whether transcriptional responses can offer a potential mechanism for hormesis, the results of this study indicated that low-dose terpenoid treatments generally elicited molecular responses (i.e., CYP450 and CP gene families) similar to those in treatments with terpenoids at high doses. However, one unanticipated finding was the upregulation of the glucose transport pathway after D-camphor exposure at a relatively low dose (Fig. 4c), suggesting a massive intake of glucose (i.e., carbohydrate biotransformed possibly from 
terpenoid-derived products) applied to larval growth (pictured in Fig. 10). Overall, hormesis is a highly generalized dose-response phenomenon and occurs frequently in various experimental designs (Calabrese, 2005; Calabrese, 2017; Kim et al., 2018), and in the context of insect-plant interactions, it deserves to be further investigated.

\section{CYP450s function in exogenous terpenoid metabolism}

Prior studies have noted that the presence of phytochemicals in host plants is considered as a major factor driving the evolution of CYP450-mediated metabolic resistance in both specialist and generalist herbivores (Alyokhin and Chen, 2017). Specialists deploy more efficient CYP450s and superior detoxification mechanisms against host-specific chemicals than generalists (Mao et al., 2006; Zhu et al., 2016). Consistent with the literature, our research provided the first evidence that several CYP450 genes belonging to the CYP6 and CYP9 subfamilies are jointly responsive to host terpenoids (i.e., EO and Dcamphor) in an emerging specialist herbivore, $P$. tsushimanus. It can be speculated that a complete metabolic pathway takes place in this beetle. Specifically, once the terpenoid molecules enter the body cell, they are immediately captured by nuclear receptors to form a complex before migrating into the nucleus. Then the complex increases the transcriptional capacity of downstream genes and achieves the massive synthesis of CYP450 enzymes engaging in the degradation of terpenoid molecules (e.g., oxidation, epoxidation, dehydrogenation, hydrolysis or reduction) and their elimination from the cell (pictured in Fig. 10). Although the transcriptional responses are expected to be directed towards the detoxification function of CYP450s, an essential next step in confirming the metabolic fate of terpenoids in $P$. tsushimanus larvae needs to be carried out. For example, using an artificial diet containing ${ }^{14} \mathrm{C}$ labelled gossypol for Heliothis virescens larvae, Rojas et al. (1992) identified that $25 \%$ of the labelled gossypol was metabolized by conjugation with six sugar molecules per mole of gossypol and excreted in the frass. Another classical case illustrated that after topically treatment with R-(+)/-S-(-)-a-pinene, the following compounds were detected using GC-MS in the hindgut extracts of the spruce bark beetle, Ips typographus: 4S-(-)-cis-verbenol, 4R-(-)-/4S-(+)-trans-verbenol, R-(+)-/S-(-)-verbenone, and 1R(-)-/1S-(+)myrtenol (Fang et al., 2021). Meanwhile, the metabolic a-pinene products were found to be sensed as aggregation pheromones by conspecifics of I. typographus. Such sequestration of putative toxic metabolites has been observed in more than 250 herbivorous insect species (Opitz and Müller, 2009). Therefore, exactly as we inferred from the issue of hormesis, in our current study, $P$. tsushimanus larvae have the capacity to sequester certain terpenoid metabolites as nutrients (e.g., carbohydrates), but further evidence is needed.

\section{CPs constitute a physical barrier against terpenoids}

The insect cuticle is composed of the polysaccharide chitin, proteins and lipids that are distributed in distinct horizontal layers, acting mainly as a physical barrier against external compounds (Moussian, 2010; Balabanidou et al., 2018). As the inner chitinous layer, the procuticle that accounts for most of the cuticular mass is thought to be closely associated with penetration resistance (i.e., cuticular resistance). Early works employing genomic analyses revealed that a variety of genes encoding structural CPs are 
constitutively deposited in the procuticle (Futahashi et al., 2008; Dittmer et al., 2015). Subsequently, there has been growing evidence that cuticular thickening attributed to the overexpression of CPs is one of the mechanisms underlying cuticular resistance (Vontas et al., 2007; Fang et al., 2015; Yahouedo et al., 2017b). In this study, transcriptome analysis in P. tsushimanus indicated that a suite of CP genes were expressed at higher levels in terpenoid treatments. Consequently, in addition to metabolic resistance, CPmediated cuticular resistance may be concomitantly recruited to decrease xenobiotic penetration (pictured in Fig. 10). It, however, remains to be further elucidated whether the elevated expression of CP proteins is consistent with the cuticle-thickening resistance mechanism. In combination with phenotypic analysis (e.g., measurement of cuticle thickness using scanning or transmission electron microscopy), a gene silencing system (e.g., RNA interference) will be a powerful tool for reversely verifying the physiological function of one or groups of CP genes. Using the above methods, Huang et al. (2018b) found that silencing of a single gene, CpCPLCG5, decreased cuticle thickness and increased pyrethroid susceptibility in the resistant strain Culex pipiens pallens.

Overall, cuticular resistance was reported in our present work in combination with metabolic resistance, suggesting an interaction between these two mechanisms. It has been widely accepted that cuticular resistance acts as a modulator that allows metabolic enzymes to work in sufficient time and consequently strengthens resistance conferred by metabolic mechanisms. Considering all these findings, we further predict that the dual terpenoid resistance mechanisms existing in this specialist are an essential precondition for the hormetic response in larval growth, ultimately contributing to the widespread successful colonization of host camphor trees.

\section{Declarations}

Ethical approval: This article does not contain any studies with human participants or vertebrates performed by any of the authors.

Funding: The research was funded by National Key R \& D Program of China (2018YFC1200400), National Science Foundation of China under Grant (31470650 and 31170606), and the Science and Technology Commission of Shanghai Municipality (18391903200).

Conflicts of interest: All authors declare no conflict of interest.

Data availability: The RNAseq datasets analyzed during the current study are available in the NCBI's Gene Expression Omnibus and are accessible through GEO Series accession number GSE182420 (https://www.ncbi.nlm.nih.)

Authors' contributions: SL and DH conceived and designed research. SL and JW conducted experiments. DH contributed new reagents and analytical tools. SL, CC and HL analyzed data. SL wrote the manuscript. All authors read and approved the manuscript.

\section{Acknowledgments}

Page $12 / 26$ 
We acknowledge Peng Su, Liang Chen, and Yanbo Sun for their assistance. Also thanks for the financial support of the Forest Station of Shanghai, Shanghai 200040, China.

\section{References}

1. Agathokleous E, Calabrese EJ (2019) Hormesis: The dose response for the 21st century: The future has arrived. Toxicology 425:152249

2. Alyokhin A, Chen YH (2017) Adaptation to toxic hosts as a factor in the evolution of insecticide resistance. Curr Opin Insect Sci 21:33-38

3. Anders S, Huber W (2010) Differential expression analysis for sequence count data. Genome biol 11:R106

4. Awolola TS, Oduola OA, Strode C et al (2009) Evidence of multiple pyrethroid resistance mechanisms in the malaria vector Anopheles gambiae sensu stricto from Nigeria. Trans R Soc Trop Med Hyg 103:1139-1145

5. Balabanidou V, Grigoraki L, Vontas J (2018) Insect cuticle: a critical determinant of insecticide resistance. Curr Opin Insect Sci 27:68-74

6. Calabrese EJ (2005) Paradigm lost, paradigm found: the re-emergence of hormesis as a fundamental dose response model in the toxicological sciences. Environ Pollut 138:379-411

7. Calabrese EJ (2017) Hormesis commonly observed in the assessment of aneuploidy in yeast. Environ Pollut 225:713-728

8. Calabreseci EJ (1999) Evidence that hormesis represents an 'overcompensation' response to a disruption in homeostasis. Ecotoxicol Environ Saf 42:135-137

9. Celorio-Mancera ML, Ahn SJ, Vogel H, Heckel DG (2011) Transcriptional responses underlying the hormetic and detrimental effects of the plant secondary metabolite gossypol on the generalist herbivore Helicoverpa armigera. BMC Genomics 12:575

10. Chen C, Zhang C, Li S et al (2020) Biological traits and life history of Pagiophloeus tsushimanus (Coleoptera: Curculionidae), a weevil pest on camphor trees in China. J For Res. https://doi.org/10.1007/s11676-020-01227-2

11. Cheng T, Wu J, Wu Y et al (2017) Genomic adaptation to polyphagy and insecticides in a major East Asian noctuid pest. Nat Ecol Evol 1:1747-1756

12. Dittmer NT, Tetreau G, Cao X et al (2015) Annotation and expression analysis of cuticular proteins from the tobacco hornworm, Manduca sexta. Insect Biochem Mol Biol 62:100-113

13. Fang F, Wang W, Zhang D et al (2015) The cuticle proteins: a putative role for deltamethrin resistance in Culex pipiens pallens. Parasitol Res 114:4421-4429

14. Fang JX, Zhang SF, Liu F et al (2021) Functional investigation of monoterpenes for improved understanding of the relationship between hosts and bark beetles. J Appl Entomol 145:303-311 
15. Fescemyer HW, Sandoya GV, Gill TA et al (2013) Maize toxin degrades peritrophic matrix proteins and stimulates compensatory transcriptome responses in fall armyworm midgut. Insect Biochem Mol Biol 43:280-291

16. Forbes VE (2000) Is hormesis an evolutionary expectation? Funct Ecol 14:12-24

17. Futahashi R, Okamoto S, Kawasaki H et al (2008) Genome-wide identification of cuticular protein genes in the silkworm, Bombyx mori. Insect Biochem Mol Biol 38:1138-1146

18. Grabherr MG, Haas BJ, Yassour M et al (2011) Full-length transcriptome assembly from RNA-Seq data without a reference genome. Nat biotechnol 29:644-652

19. Heckel DG (2014) Insect detoxification and sequestration strategies. Annu Plant Rev 47:77-114

20. Heidel-Fischer HM, Vogel H (2015) Molecular mechanisms of insect adaptation to plant secondary compounds. Curr Opin Insect Sci 8:8-14

21. Hrycay EG, Bandiera SM (2015) Monooxygenase, peroxidase and peroxygenase properties and reaction mechanisms of cytochrome P450 enzymes. (Hrycay EG, Bandiera SM (eds)), pp 1-61. Springer International Publishing, Cham

22. Huang Y, Liao M, Yang Q et al (2018a) Transcriptome profiling reveals differential gene expression of detoxification enzymes in Sitophilus zeamais responding to terpinen-4-ol fumigation. Pestic Biochem Physiol 149:44-53

23. Huang Y, Guo Q, Sun $X$ et al (2018b) Culex pipiens pallens cuticular protein CPLCG5 participates in pyrethroid resistance by forming a rigid matrix. Parasit Vectors 11:6

24. Jager T, Barsi A, Ducrot V (2013) Hormesis on life-history traits: is there such thing as a free lunch? Ecotoxicology 22:263-270

25. Kim SA, Lee YM, Choi JY, Jacobs DJ, Lee DH (2018) Evolutionarily adapted hormesis-inducing stressors can be a practical solution to mitigate harmful effects of chronic exposure to low dose chemical mixtures. Environ Pollut 233:725-734

26. Kliot A, Kontsedalov S, Ramsey JS, Jander G, Ghanim M (2014) Adaptation to nicotine in the facultative tobacco-feeding hemipteran Bemisia tabaci. Pest Manage Sci 70:1595-1603

27. Langfelder P, Horvath S (2008) WGCNA: an R package for weighted correlation network analysis. BMC Bioinf 9:559

28. Li B, Dewey CN (2011) RSEM: accurate transcript quantification from RNA-Seq data with or without a reference genome. BMC Bioinf 12:323

29. Li Q, Sun Z, Shi Q et al (2019) RNA-Seq analyses of midgut and fat body tissues reveal the molecular mechanism underlying Spodoptera litura resistance to tomatine. Front Physiol 10:8

30. Li SY, Chen C, Jia ZY et al (2021) Offspring performance and female preference of Pagiophloeus tsushimanus (coleoptera: curculionidae) on three Lauraceae tree species: a potential risk of host shift caused by larval experience. J Appl Entomol 145:530-542

31. Liao M, Xiao J, Zhou L et al (2016) Insecticidal activity of Melaleuca alternifolia essential oil and RNA-Seq analysis of Sitophilus zeamais transcriptome in response to oil fumigation. PLoS One 
11:e167748

32. Lu K, Song Y, Zeng R (2021) The role of cytochrome P450-mediated detoxification in insect adaptation to xenobiotics. Curr Opin Insect Sci 43:103-107

33. Magalhaes DM, Borges M, Laumann RA et al (2020) Inefficient weapon-the role of plant secondary metabolites in cotton defence against the boll weevil. Planta 252:94

34. Mao W, Rupasinghe S, Zangerl AR, Schuler MA, Berenbaum MR (2006) Remarkable substratespecificity of CYP6AB3 in Depressaria pastinacella, a highly specialized caterpillar. Insect Mol Biol 15:169-179

35. Maurya AK, Patel RC, Frost CJ (2020) Acute toxicity of the plant volatile indole depends on herbivore specialization. J Pest Sci 93:1107-1117

36. Mossa AH (2016) Green pesticides: essential oils as biopesticide in insect-pest management. J Environ Sci Technol 9:354-378

37. Moussian B (2010) Recent advances in understanding mechanisms of insect cuticle differentiation. Insect Biochem Mol Biol 40:363-375

38. Müller C, Vogel H, Heckel DG (2017) Transcriptional responses to short-term and long-term host plant experience and parasite load in an oligophagous beetle. Mol Ecol 26:6370-6383

39. Nauen R, Zimmer CT, Vontas J (2021) Heterologous expression of insect P450 enzymes that metabolize xenobiotics. Curr Opin Insect Sci 43:78-84

40. Nkya TE, Poupardin R, Laporte F et al (2014) Impact of agriculture on the selection of insecticide resistance in the malaria vector Anopheles gambiae: a multigenerational study in controlled conditions. Parasit Vectors 7:480

41. Ntalli N, Koliopoulos G, Giatropoulos A, Menkissoglu-Spiroudi U (2019) Plant secondary metabolites against arthropods of medical importance. Phytochem Rev 18:1255-1275

42. Opitz SE, W, Müller C (2009) Plant chemistry and insect sequestration. Chemoecology 19:117-154

43. Petschenka G, Agrawal AA (2016) How herbivores coopt plant defenses: natural selection, specialization, and sequestration. Curr Opin Insect Sci 14:17-24

44. Puinean AM, Foster SP, Oliphant L et al (2010) Amplification of a cytochrome P450 gene is associated with resistance to neonicotinoid insecticides in the aphid Myzus persicae. PLoS Genet 6:e1000999

45. Ramsey JS, Elzinga DA, Sarkar P et al (2014) Adaptation to nicotine feeding in Myzus persicae. J Chem Ecol 40:869-877

46. Rojas MG, Stipanovic RD, Williams HJ, Vinson SB (1992) Metabolism of Gossypol by Heliothis virescens (F.) (Lepidoptera: Noctuidae). Environ Entomol 21:518-526

47. Roy A, Walker WB, Vogel H et al (2016) Diet dependent metabolic responses in three generalist insect herbivores Spodoptera spp. Insect Biochem Mol Biol 71:91-105

48. Salehipourshirazi G, Bruinsma K, Ratlamwala H et al (2020) The generalist herbivore Tetranychus urticae (Koch) adapts to novel plant hosts through rapid evolution of metabolic resistance. BioRxiv. 
https://doi.org/10.1101/2020.02.26.966481

49. Schmittgen TD, Livak KJ (2008) Analyzing real-time PCR data by the comparative C(T) method. Nat Protoc 3:1101-1108

50. Schweizer F, Heidel-Fischer H, Vogel H, Reymond P (2017) Arabidopsis glucosinolates trigger a contrasting transcriptomic response in a generalist and a specialist herbivore. Insect Biochem Mol Biol 85:21-31

51. Shannon P, Markiel A, Ozier O et al (2003) Cytoscape: a software environment for integrated models of biomolecular interaction networks. Genome Res 13:2498-2504

52. Singh VK, Mangalam AK, Dwivedi S, Naik S (1998) Primer premier: program for design of degenerate primers from a protein sequence. Biotechniques 24:318-319

53. Soto IM, Carreira VP, Corio C et al (2014) Differences in tolerance to host cactus alkaloids in Drosophila koepferae and D. buzzatii. PloS One 9:e88370

54. Southam CM, Erlich J (1943) Effects of extracts of western red-cedar heartwood on certain wooddecaying fungi in culture. Phytopathology 33:517-524

55. Stahl E, Hilfiker O, Reymond P (2018) Plant-arthropod interactions: who is the winner? Plant J 93:703-728

56. Stebbing AR (1998) A theory for growth hormesis. Mutat Res 403:249-258

57. Stipanovic RD, Lopez JJ, Dowd MK, Puckhaber LS, Duke SE (2006) Effect of racemic and (+)- and (-)gossypol on the survival and development of Helicoverpa zea larvae. J Chem Ecol 32:959-968

58. Stipanovic RD, Lopez JJ, Dowd MK, Puckhaber LS, Duke SE (2008) Effect of racemic, (+)- and (-)gossypol on survival and development of Heliothis virescens larvae. Environ Entomol 37:1081-1085

59. Tangtrakulwanich K, Reddy GVP (2014) Development of insect resistance to plant Biopesticides: an overview. (Singh D (ed)), pp 47-62. Springer India, New Delhi

60. Todd EV, Black MA, Gemmell NJ (2016) The power and promise of RNA-seq in ecology and evolution. Mol Ecol 25:1224-1241

61. Vaiserman AM (2010) Hormesis, adaptive epigenetic reorganization, and implications for human health and longevity. Dose-Response 8:16-21

62. Vandenhole M, Dermauw W, Van Leeuwen T (2021) Short term transcriptional responses of P450s to phytochemicals in insects and mites. Curr Opin Insect Sci 43:117-127

63. Vontas J, David JP, Nikou D et al (2007) Transcriptional analysis of insecticide resistance in Anopheles stephensi using cross-species microarray hybridization. Insect Mol Biol 16:315-324

64. Wybouw N, Zhurov V, Martel C et al (2015) Adaptation of a polyphagous herbivore to a novel host plant extensively shapes the transcriptome of herbivore and host. Mol Ecol 24:4647-4663

65. Yahouedo GA, Chandre F, Rossignol M et al (2017) Contributions of cuticle permeability and enzyme detoxification to pyrethroid resistance in the major malaria vector Anopheles gambiae. Sci Rep 7:11091 
66. Yu Y, Shen G, Zhu H, Lu Y (2010) Imidacloprid-induced hormesis on the fecundity and juvenile hormone levels of the green peach aphid Myzus persicae (Sulzer). Pestic Biochem Physiol 98:238242

67. Zhang X, Kang X, Wu H et al (2018) Transcriptome-wide survey, gene expression profiling and exogenous chemical-induced transcriptional responses of cytochrome P450 superfamily genes in Migratory locust (Locusta migratoria). Insect Biochem Mol Biol 100:66-77

68. Zhu F, Moural TW, Nelson DR, Palli SR (2016) A specialist herbivore pest adaptation to xenobiotics through up-regulation of multiple Cytochrome P450s. Sci Rep 6:20421

\section{Figures}

(a)

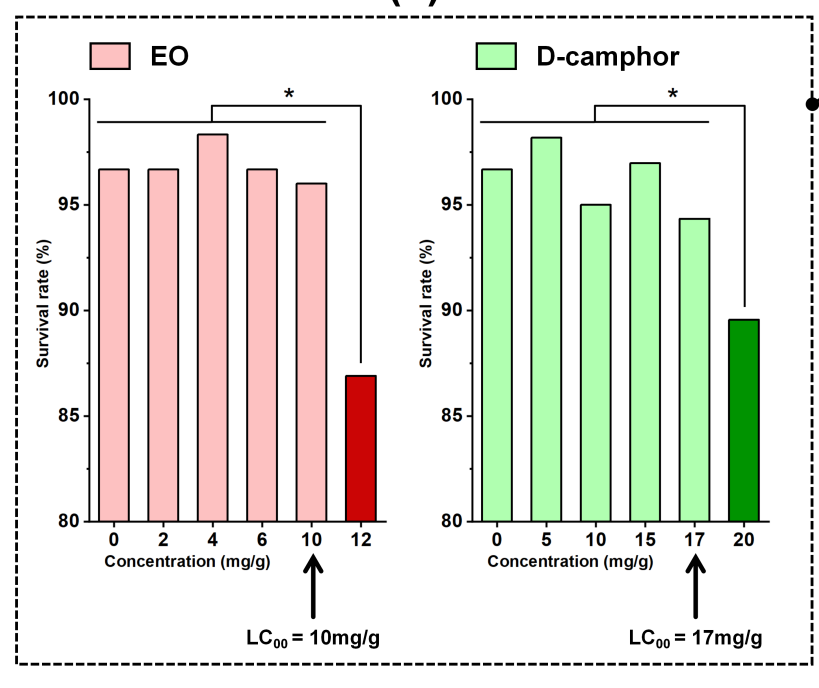

(b)

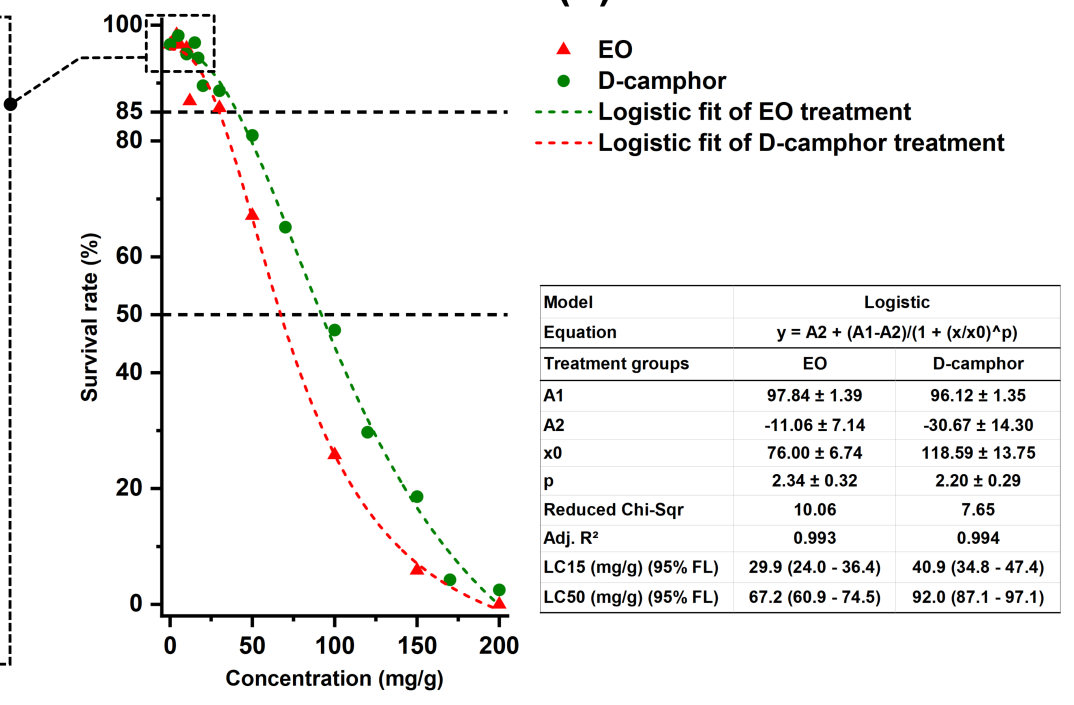

\section{Figure 1}

Concentration-response curves of Pagiophloeus tsushimanus larvae to camphor oil (EO) and D-camphor. a is the maximum nonlethal concentration (LCOO) determined in the feeding bioassays at low concentrations. $b$ is the lethal concentrations (i.e., LC15 and LC50) calculated using quadratic logistic regression. Asterisks indicate that the survival rates at different concentrations are significantly different according to the chi-square test. ${ }^{*} P<0.05,{ }^{*} \mathrm{P}<0.01,{ }^{\star *} \mathrm{P}<0.001$, NS, not significant. 
(a)
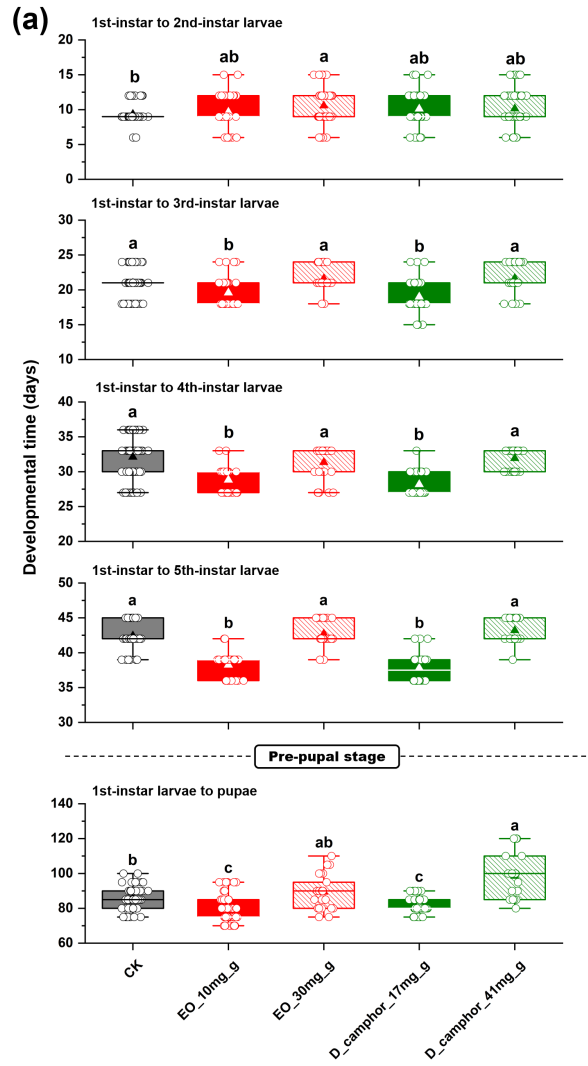

(d)

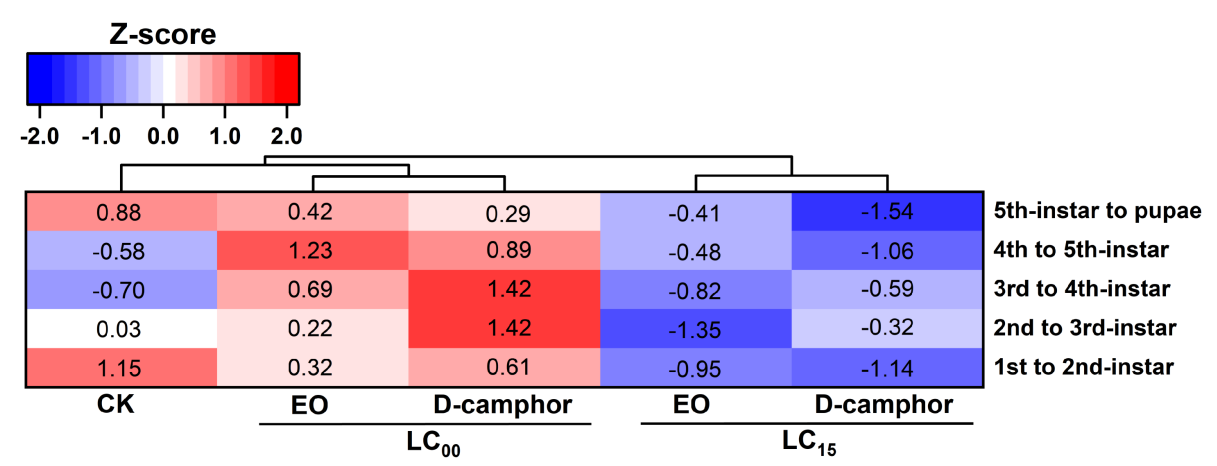

(b)
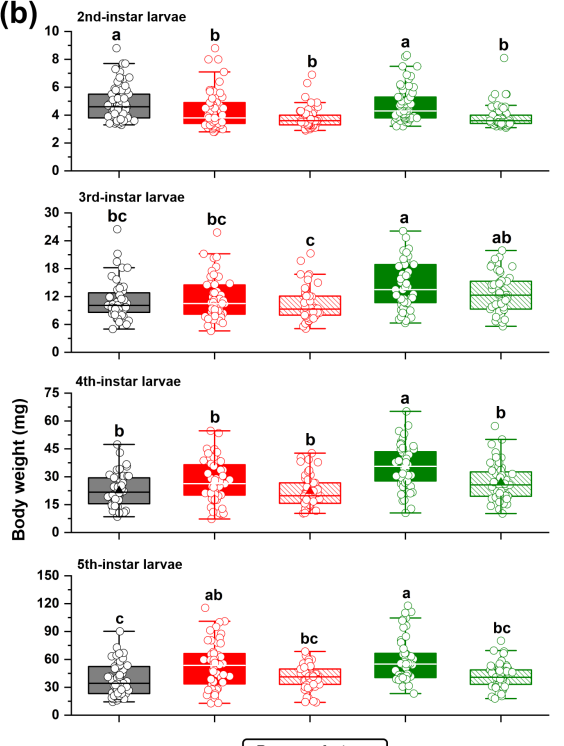

Pre-pupal stage
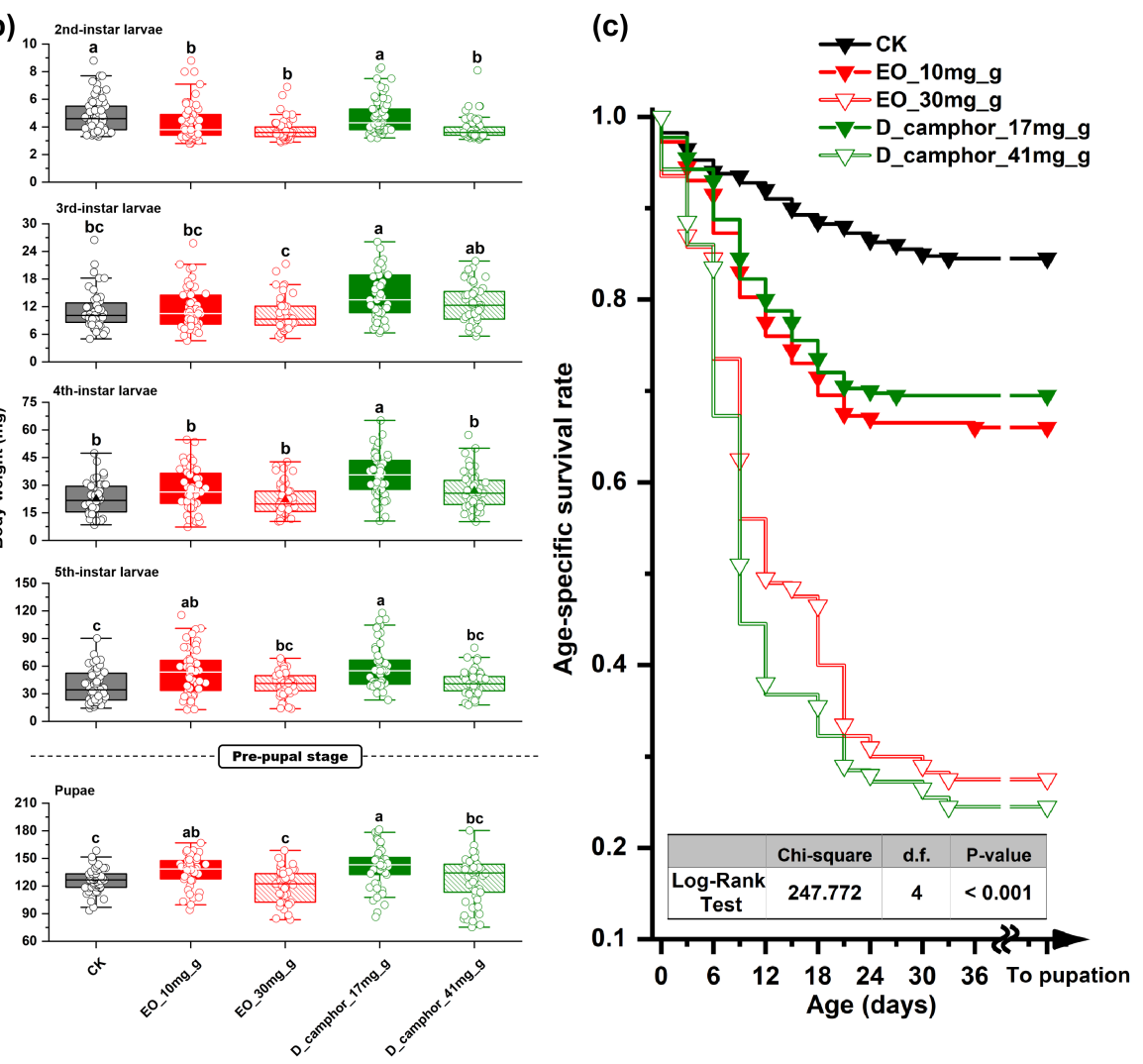

\section{Figure 2}

Developmental parameters of P. tsushimanus larvae fed terpenoid-infused diets. a, b \& c represent the developmental time, body weight and age-specific survival rate of P. tsushimanus larvae treated with EO_10mg_g, EO_30mg_g, D-camphor_17mg_g and D-camphor_41mg_g, respectively. The line and triangles inside the boxes indicate the medians and means, respectively; the height of the boxes indicates the first and third quadrilles, and the whiskers indicate $1.5 \times$ the interquartile data range. Different lowercase letters indicate significant differences among treatment groups according to a Kruskal-Wallis ANOVA test at $\mathrm{P}<0.05$. $\mathrm{d}$ Heatmap of the relative performance index (RPI) between the terpenoid treatment and control groups. Red bars indicate better biological performance, while blue bars indicate poorer biological performance. 
(a)

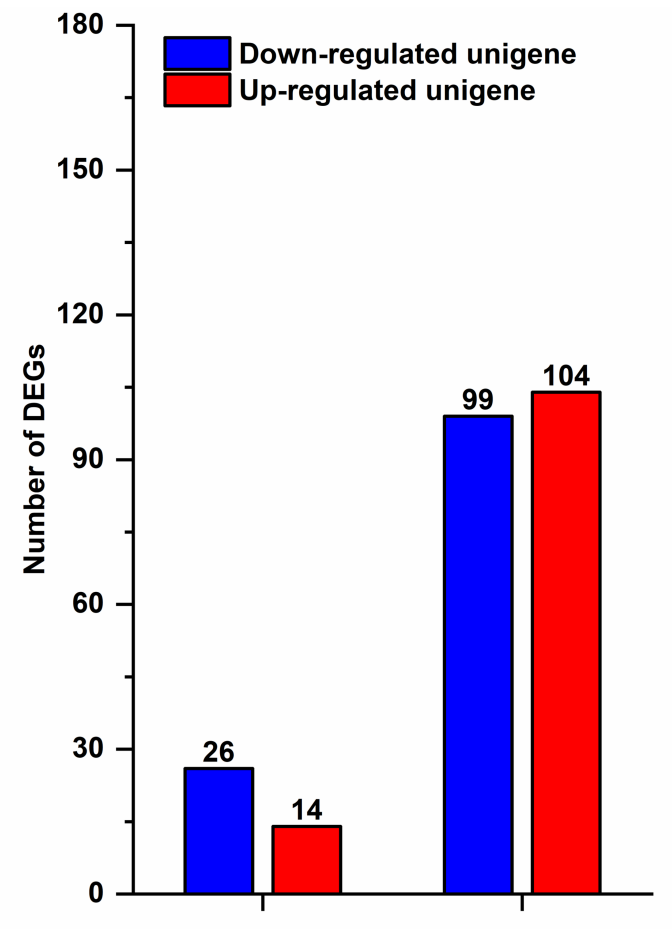

(b)

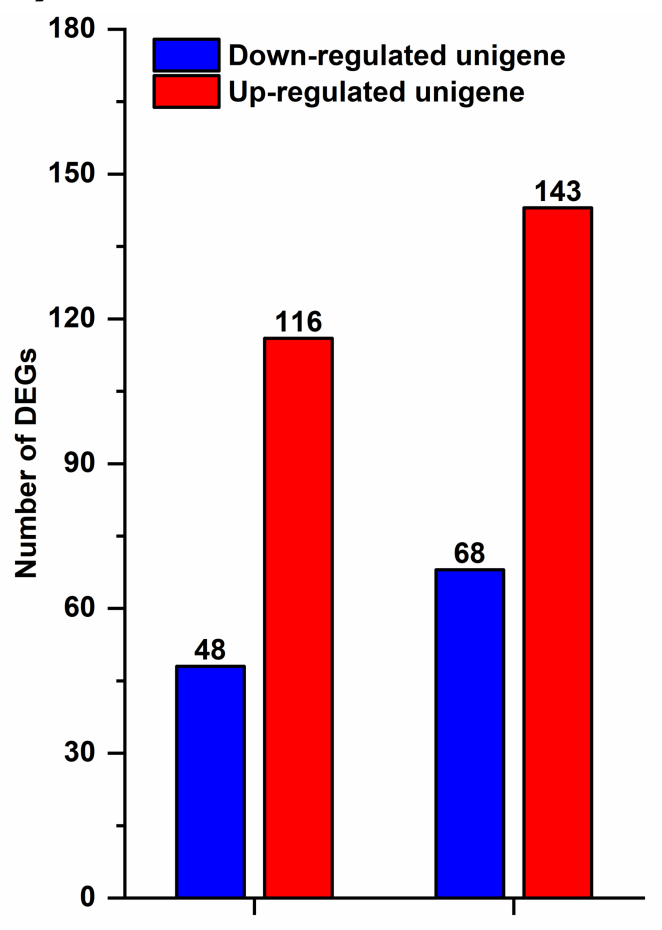

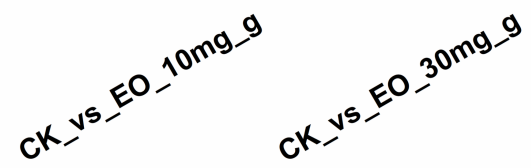

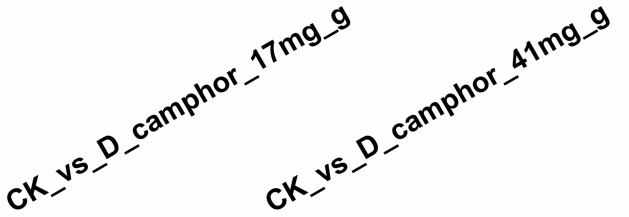

(c)

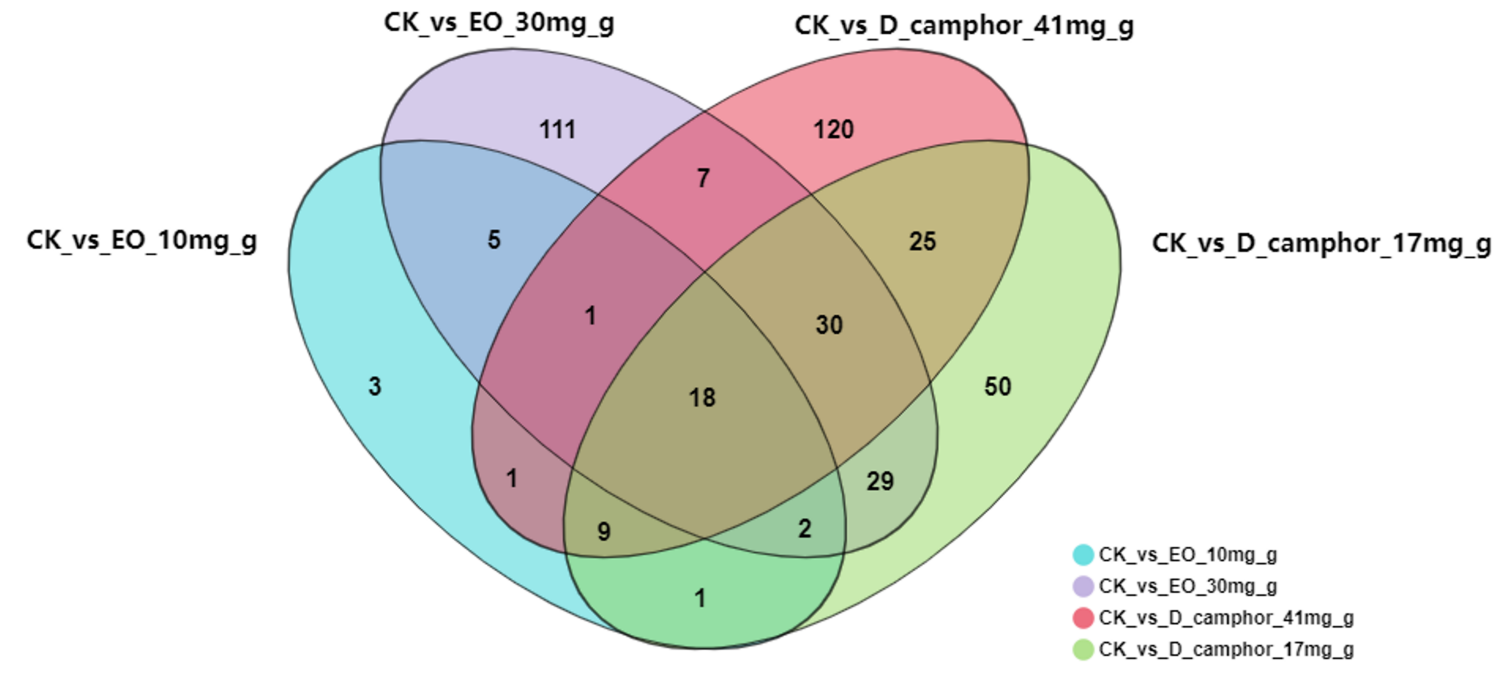

Figure 3

Basic information of differentially expressed genes (DEGs) in P. tsushimanus larvae subjected to terpenoid treatments. a summarizes the number of up- and downregulated DEGs in various treatments. $b$ is a Venn diagram depicting the distribution of DEGs. P-value $<0.05$ (i.e., false discovery rate $\leq 0.01$ ) and $|\log 2 \mathrm{FC}| \geq 1$ were used as the thresholds to identify DEGs between the terpenoid treatment and control groups. 


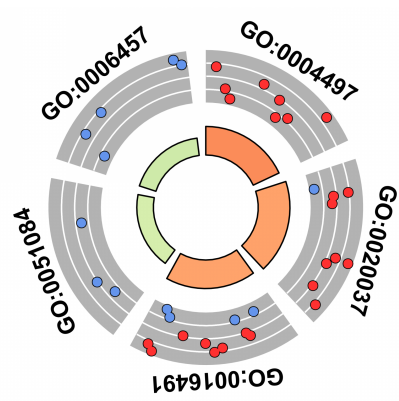

(a)
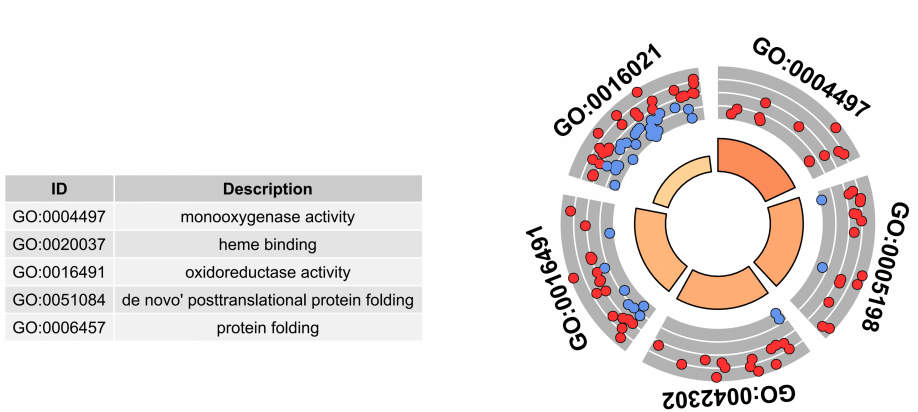

(b)

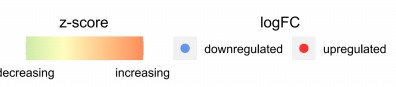

(c)
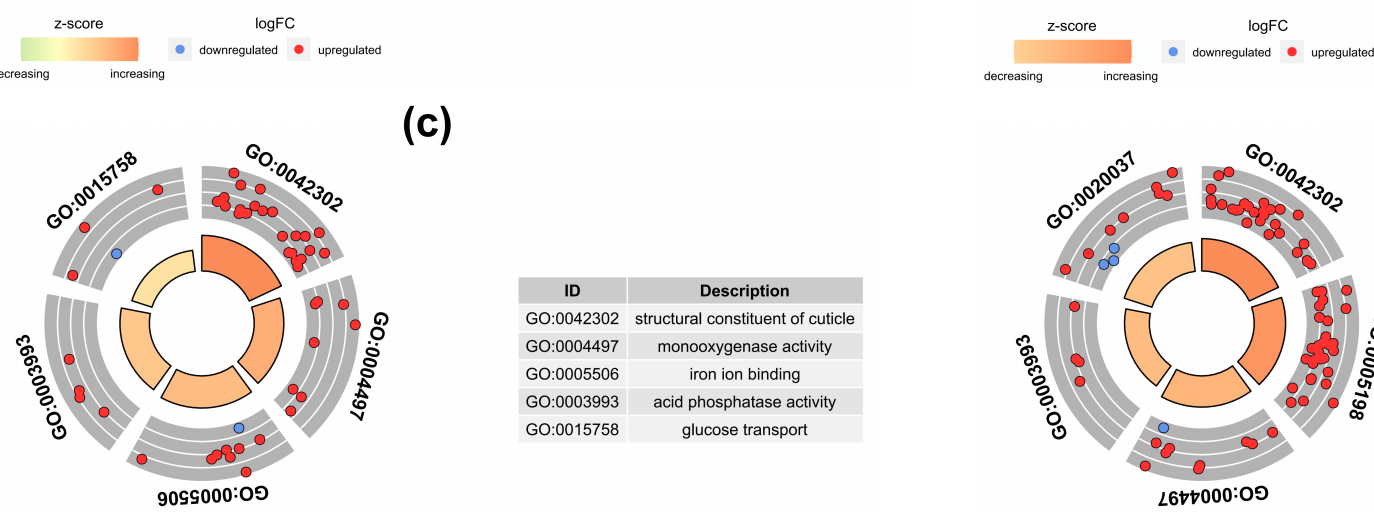

(d)
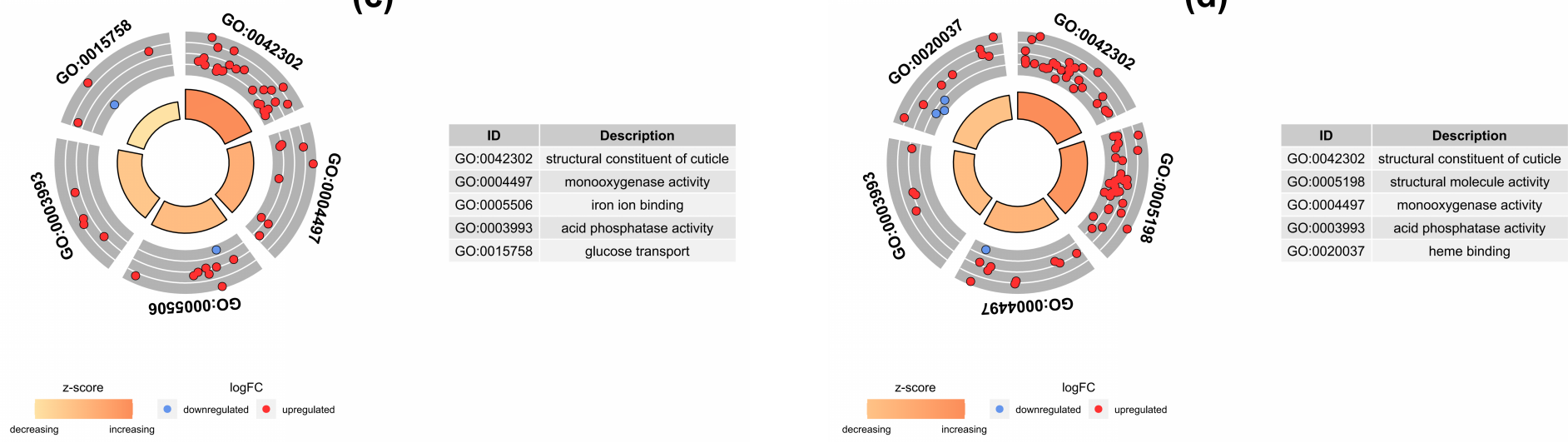

\section{Figure 4}

Top five significantly functionally enriched Gene Ontology (GO) terms among DEGs of P. tsushimanus larvae treated with EO_10mg_g (a), EO_30mg_g (b), D-camphor_17mg_g (c), and D-camphor_41mg_g (d). Red, white, and green sectors indicate that the gene-set expression levels of terpenoid treatments were higher than, similar to, and lower than those of the control group, respectively. 
(a)

\section{GO: 0004497}

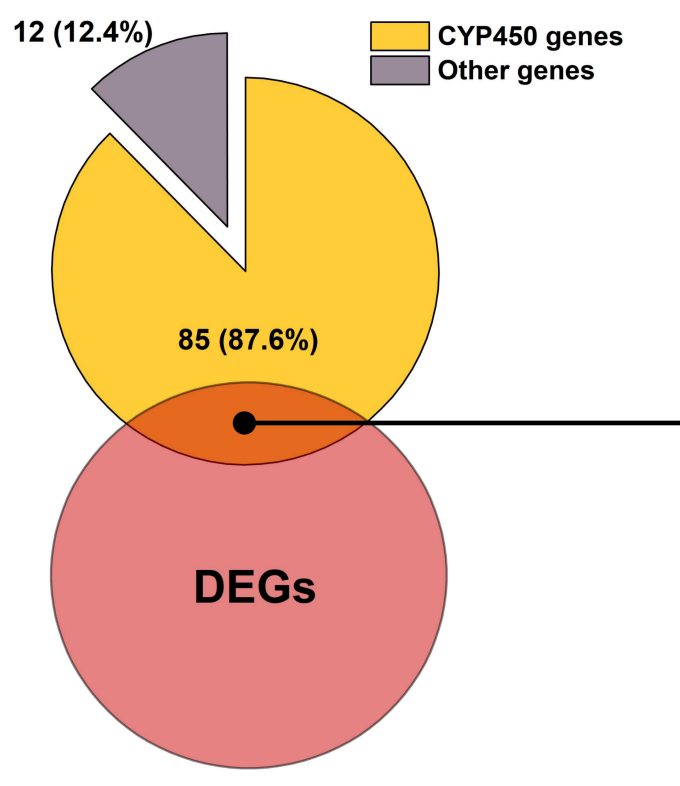

(b)
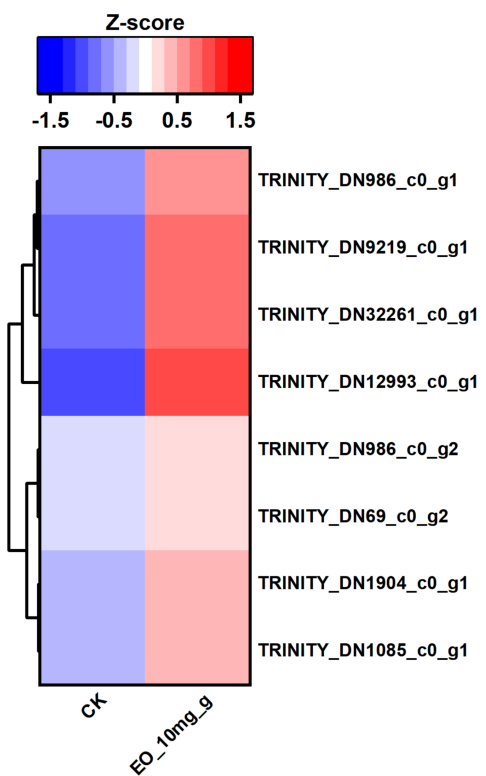

(d)

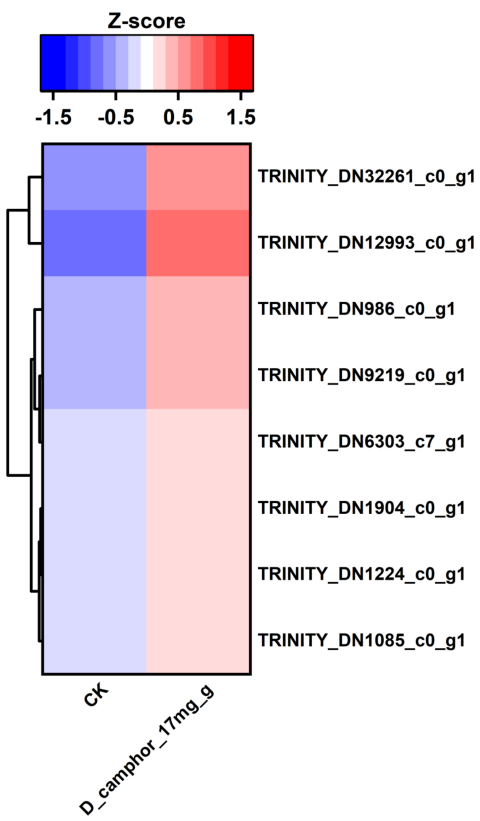

(c)
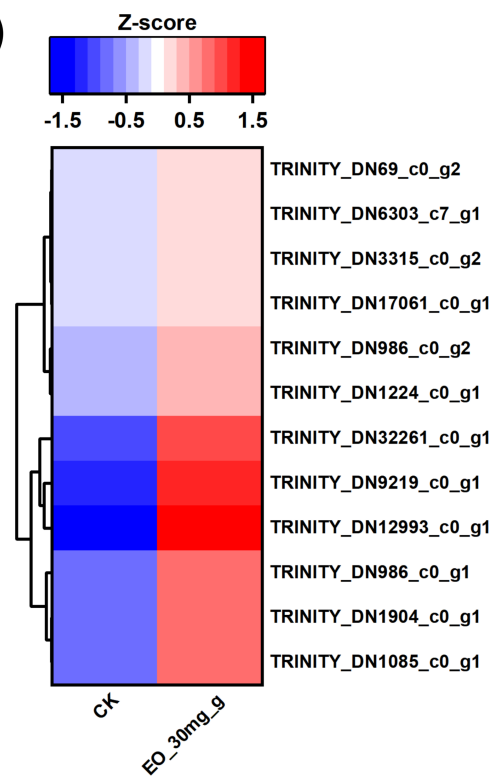

(e)

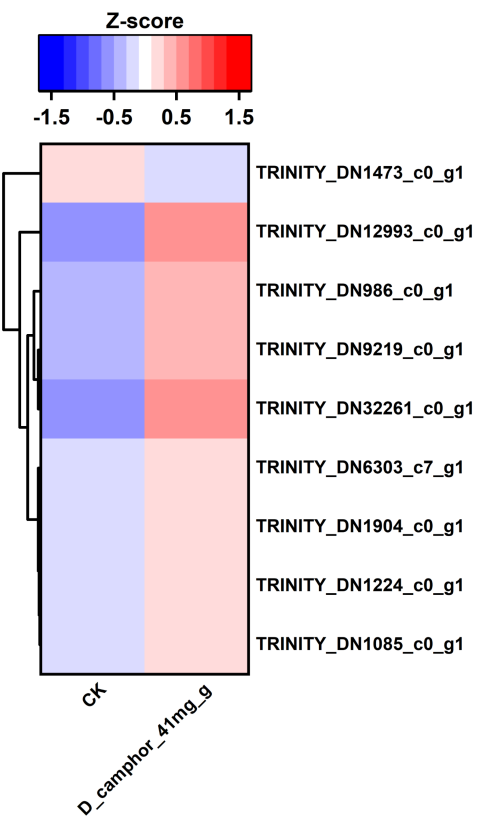

Figure 5

Identification of key functional genes based on DEGs and GO enrichment analysis - Cytochrome P450 monooxygenases (CYP450s). $a$ is a Venn diagram depicting the assignment of CYP450 genes to the 'monooxygenase activity' pathway (GO: 0004497). b, c \& d are heatmaps and hierarchical clustering graphs of the CYP450 genes that were differentially expressed between the terpenoid treatment and control groups. Red bars indicate upregulated genes, while blue bars indicate downregulated genes. 
(a)

\section{GO: 0042302}

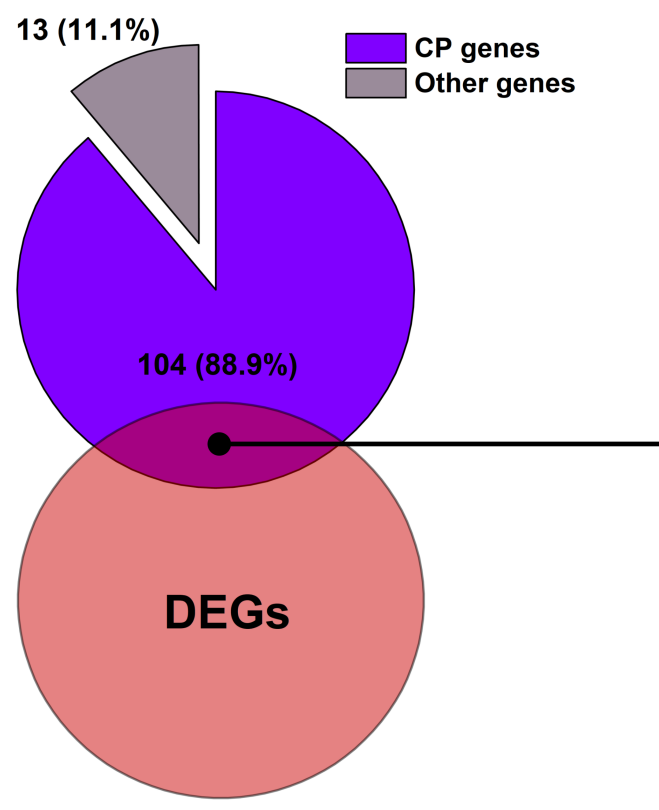

(b)
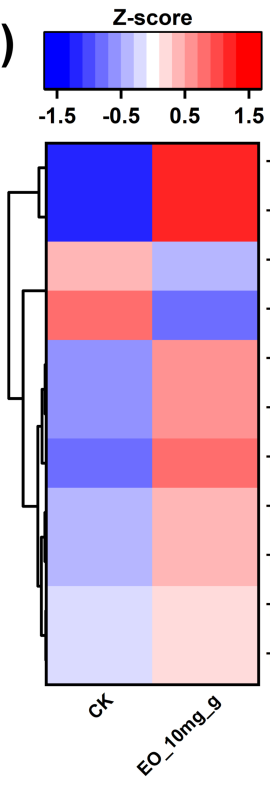

TRINITY_DN3394_C0_g1

TRINITY_DN24856_c0_g1

TRINITY_DN3873_c0_g1

TRINITY_DN17856_c0_g1

TRINITY_DN5193_c0_g1

TRINITY_DN39059_C0_g1

TRINITY_DN153_c0_g1

TRINITY_DN42053_c0_g

TRINITY_DN2281_c0_g1

TRINITY_DN1984_C0_g1

TRINITY_DN1246_C0_g1

(d)
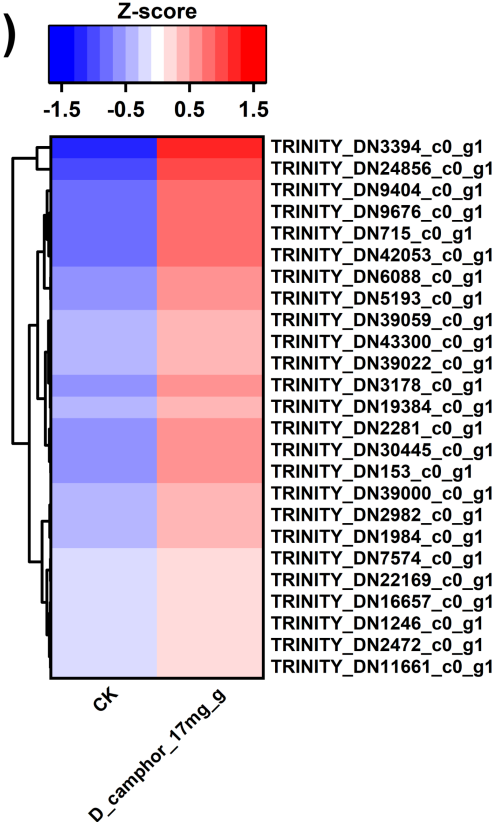

(c)
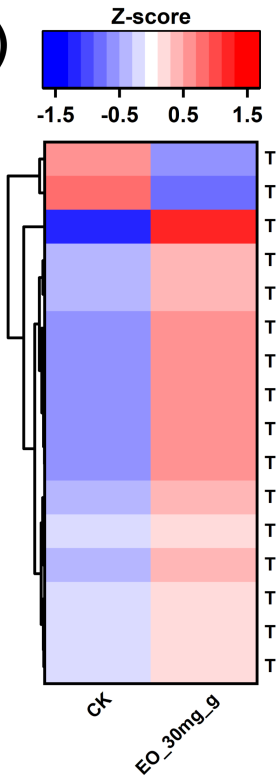

TRINITY_DN3873_c0_g1 TRINITY_DN17856_c0_g1 TRINITY_DN24856_C0_g1 TRINITY_DN39059_c0_g1 TRINITY_DN3178_c0_g1 TRINITY_DN2281_c0_g1 TRINITY_DN42053_c0_g1 TRINITY_DN3394_C0_g1 TRINITY_DN5193_c0_g1 TRINITY_DN153_C0_g1 TRINITY_DN1984_c0_g1 TRINITY_DN2982_c0_g1 TRINITY_DN19384_C0_g1 TRINITY_DN22169_C0_g1 TRINITY_DN16657_c0_g1 TRINITY_DN1246_c0_g1

(e)
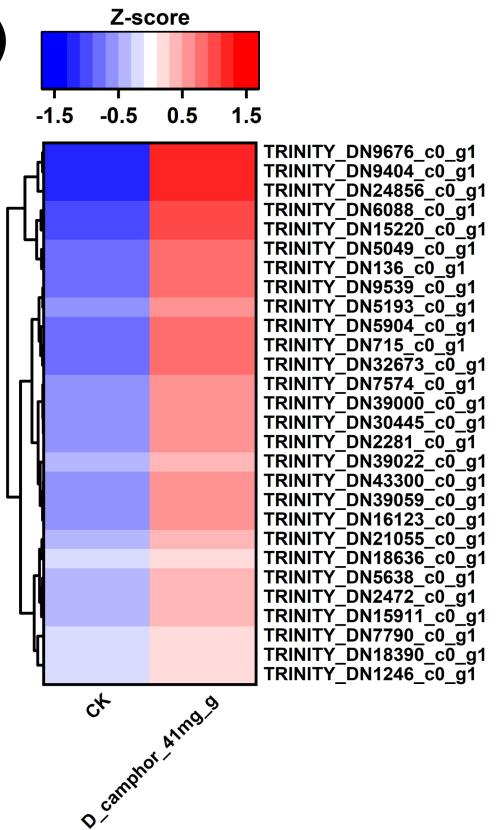

Figure 6

Identification of key functional genes based on DEGs and GO enrichment analysis - Cuticular proteins (CPs). a is a Venn diagram depicting the assignment of CP genes to the 'structural constituent of cuticle' pathway (GO: 0042302). b, c \& d are heatmaps and hierarchical clustering graphs of the CP genes that were differentially expressed between the terpenoid treatment and control groups. Red bars indicate upregulated genes, while blue bars indicate downregulated genes. 
(a)

Key modules

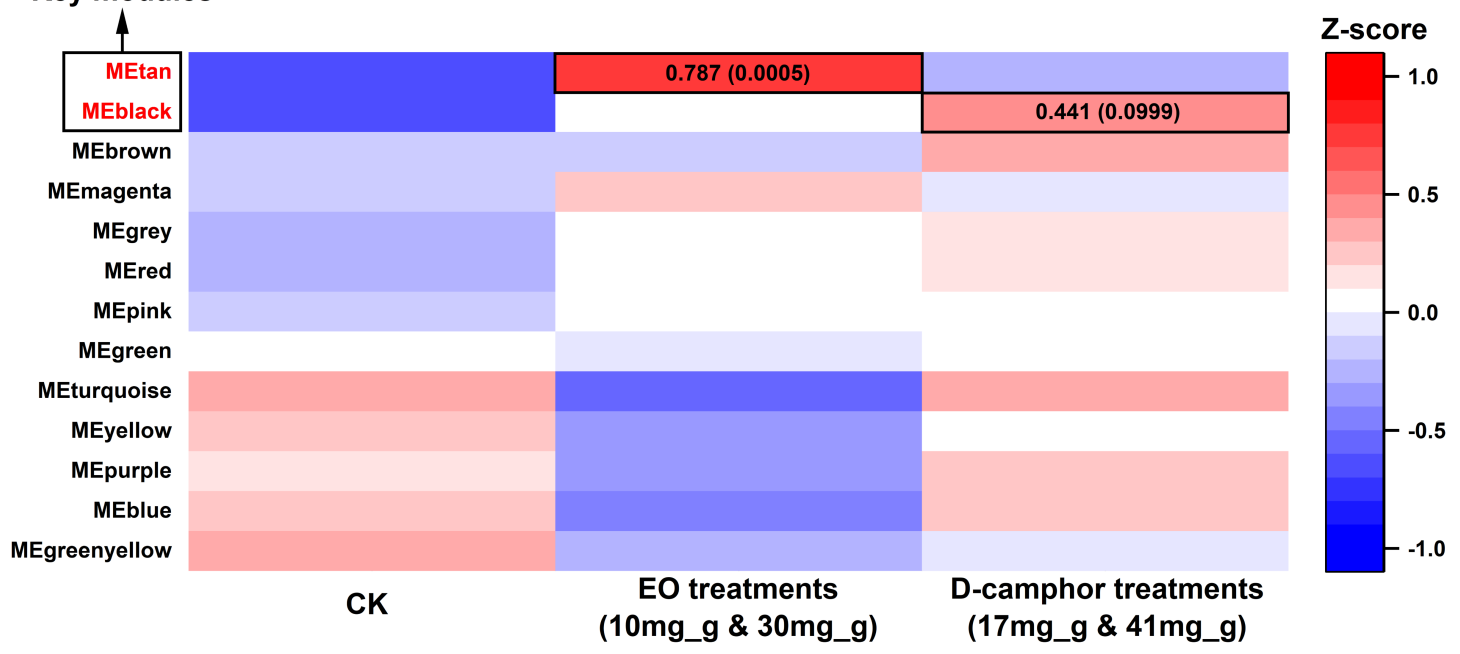

(b)

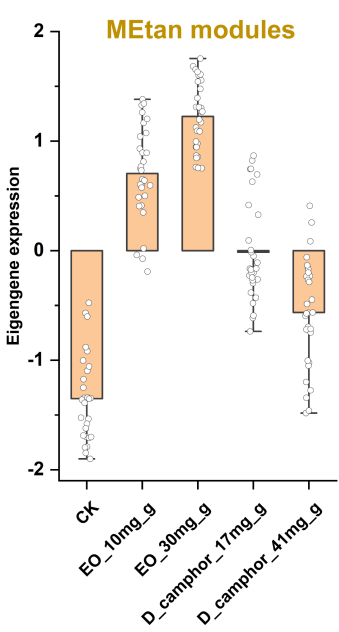

(e)

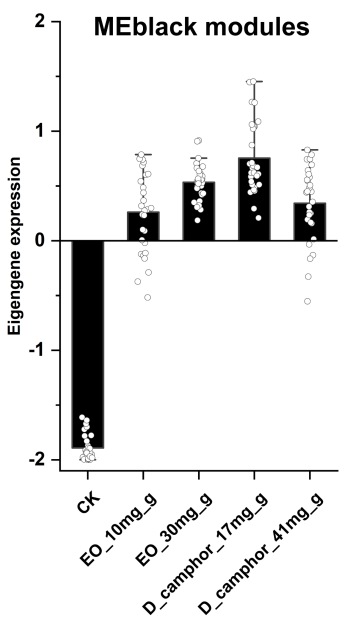

(c)

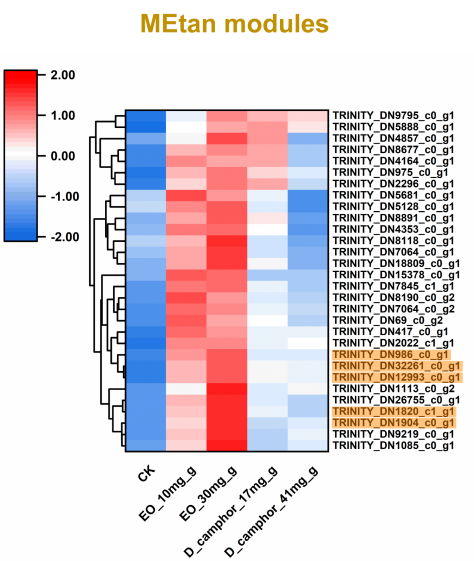

(f)

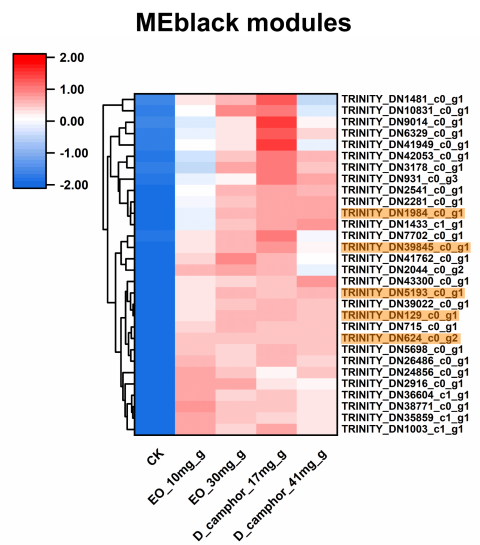

(d)

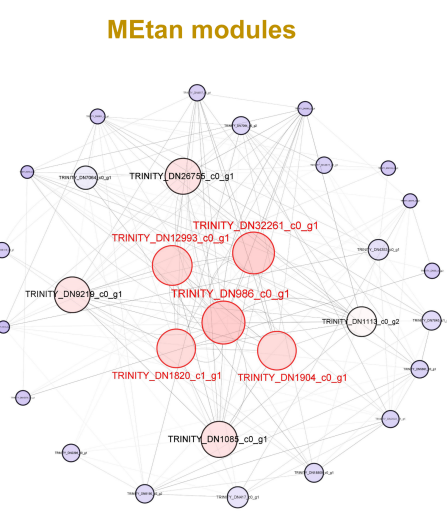

(g)

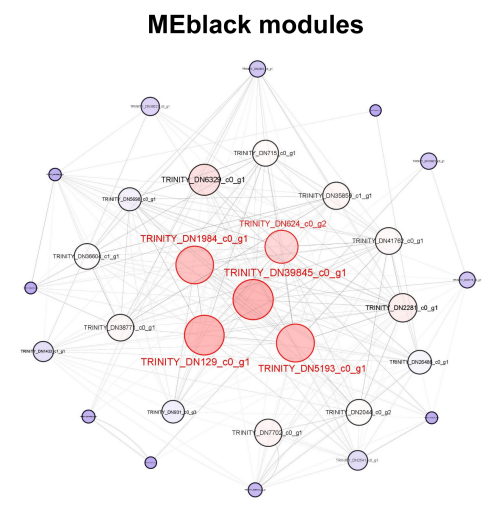

\section{Figure 7}

Identification of hub genes based on WGCNA. a is module-treatment correlation analysis. The colour of each cell at the row-column intersection indicates the correlation coefficient between the module and treatment group. MEtan and MEblack modules were selected as key modules with the highest degree of correlation with EO and D-camphor treatment, respectively. b \& e are gene expression patterns (mean \pm SD) in the MEtan and MEblack modules, respectively. c \& $f$ are heatmaps and hierarchical clustering 
graphs of the 30 most highly connected hub genes in the MEtan and MEblack modules, respectively. Red bars indicate upregulated genes, while blue bars indicate downregulated genes. $\mathrm{d} \& \mathrm{~g}$ are co-expression networks of the 30 most highly connected hub genes in the MEtan and MEblack modules, respectively.

(a)
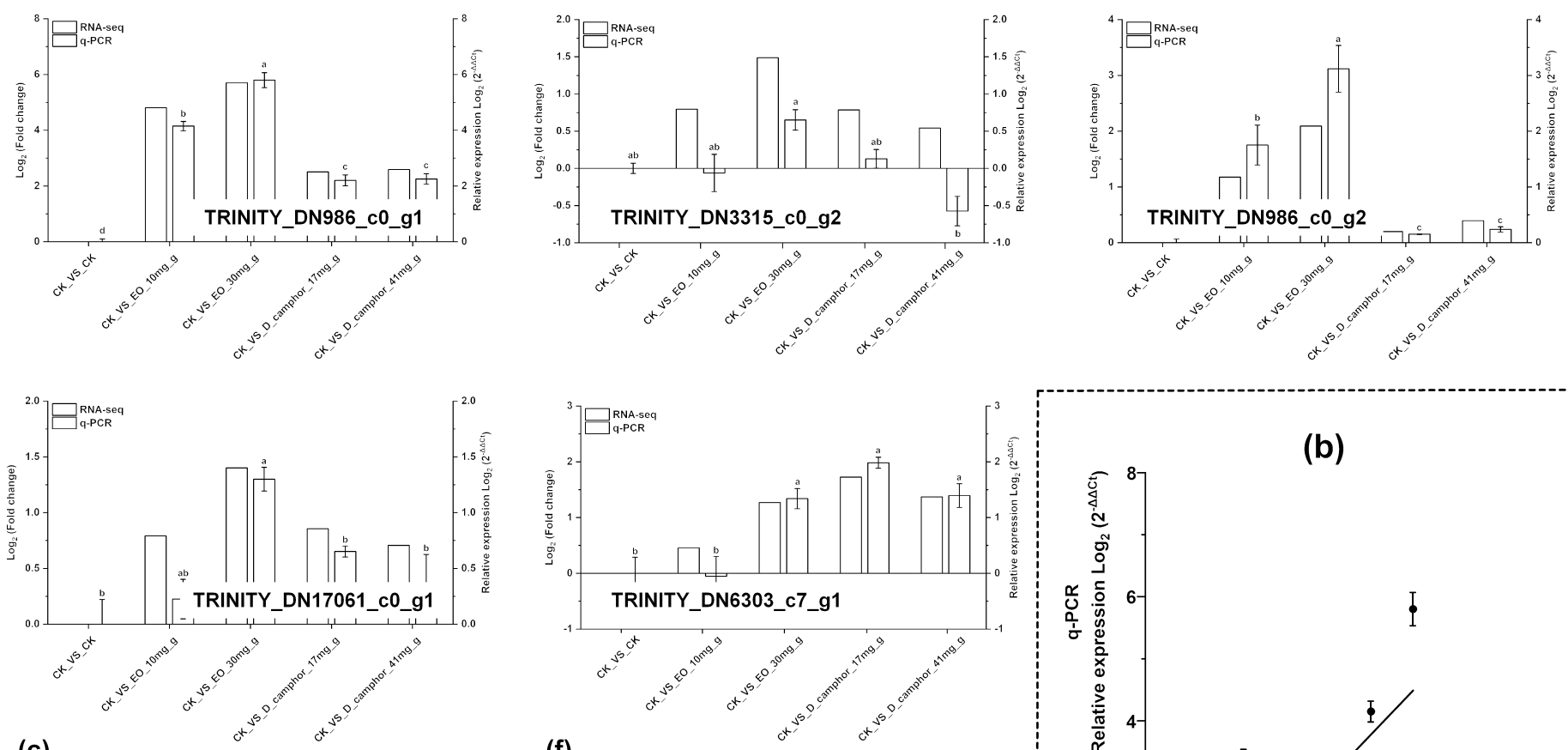

(†)

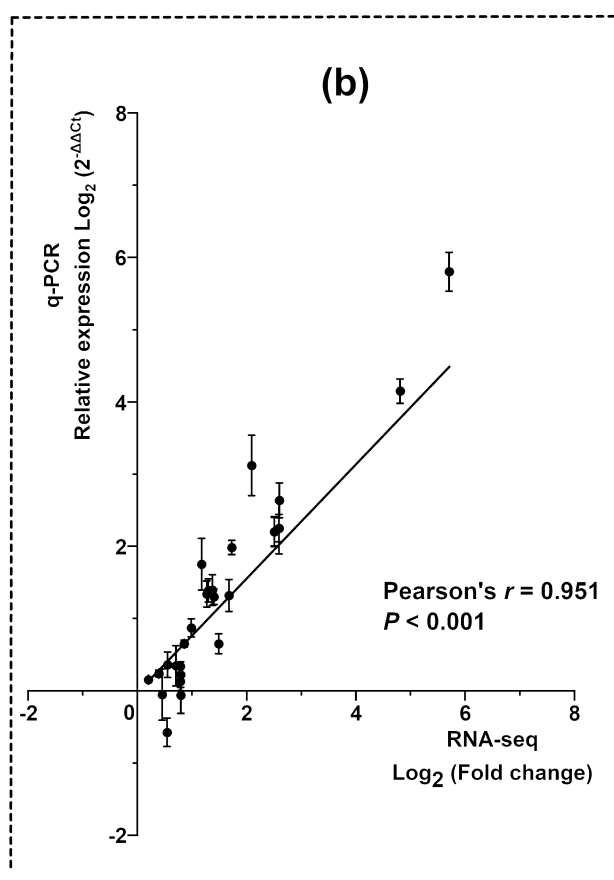

\section{Figure 8}
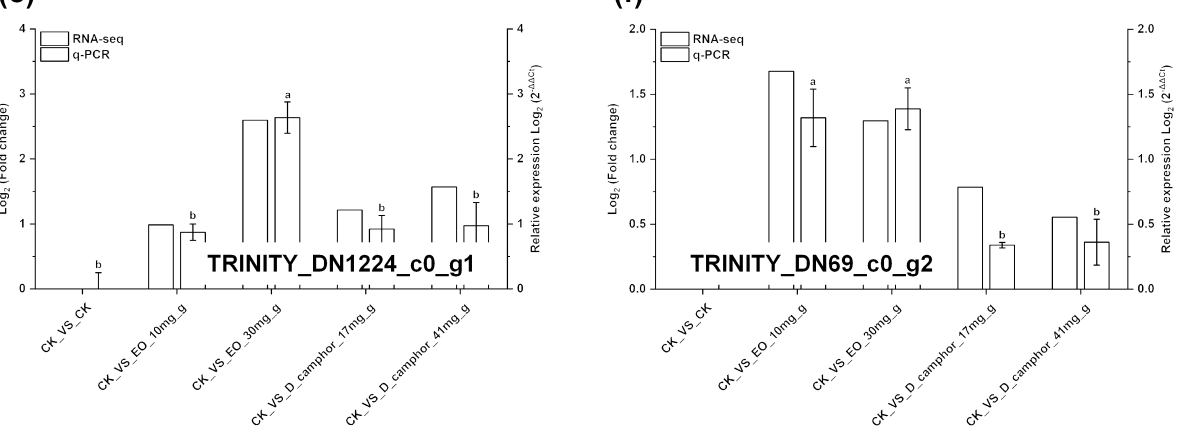

Relative expression levels of seven DEGs encoding CYP450s in P. tsushimanus treated with EO and Dcamphor. a is comparative analysis of RNA-Seq and RT-qPCR results. $b$ is the correlation between RNASeq and RT-qPCR results determined using the Pearson correlation coefficient. 
(a)
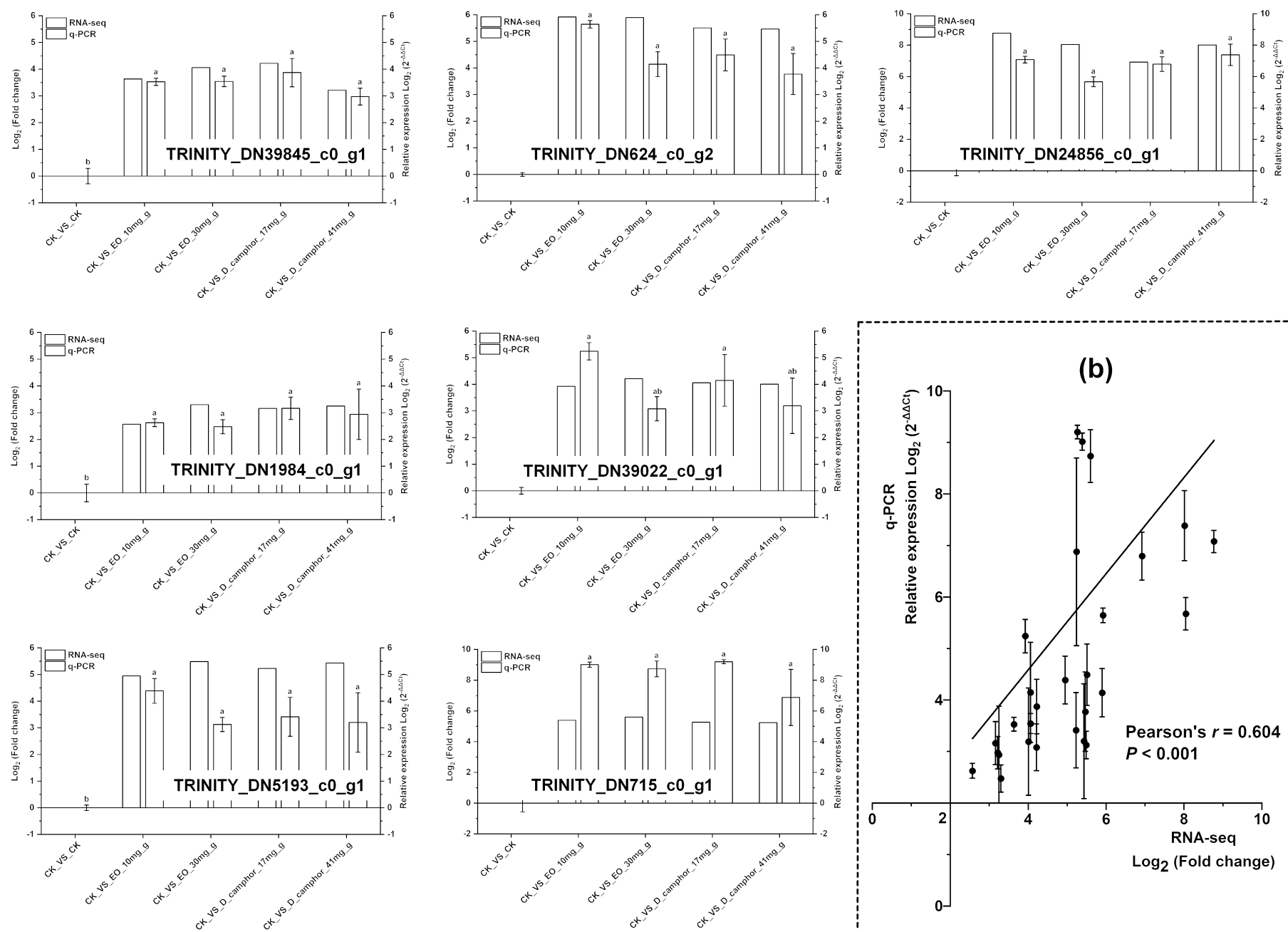

Figure 9

Relative expression levels of seven DEGs encoding CPs in P. tsushimanus treated with EO and Dcamphor. a is comparative analysis of RNA-Seq and RT-qPCR results. Different lowercase letters indicate significant differences among treatment groups according to one-way ANOVA followed by Tukey's HSD test at $P<0.05$. $b$ is the correlation between RNA-Seq and RT-qPCR results obtained using Pearson correlation coefficient. 

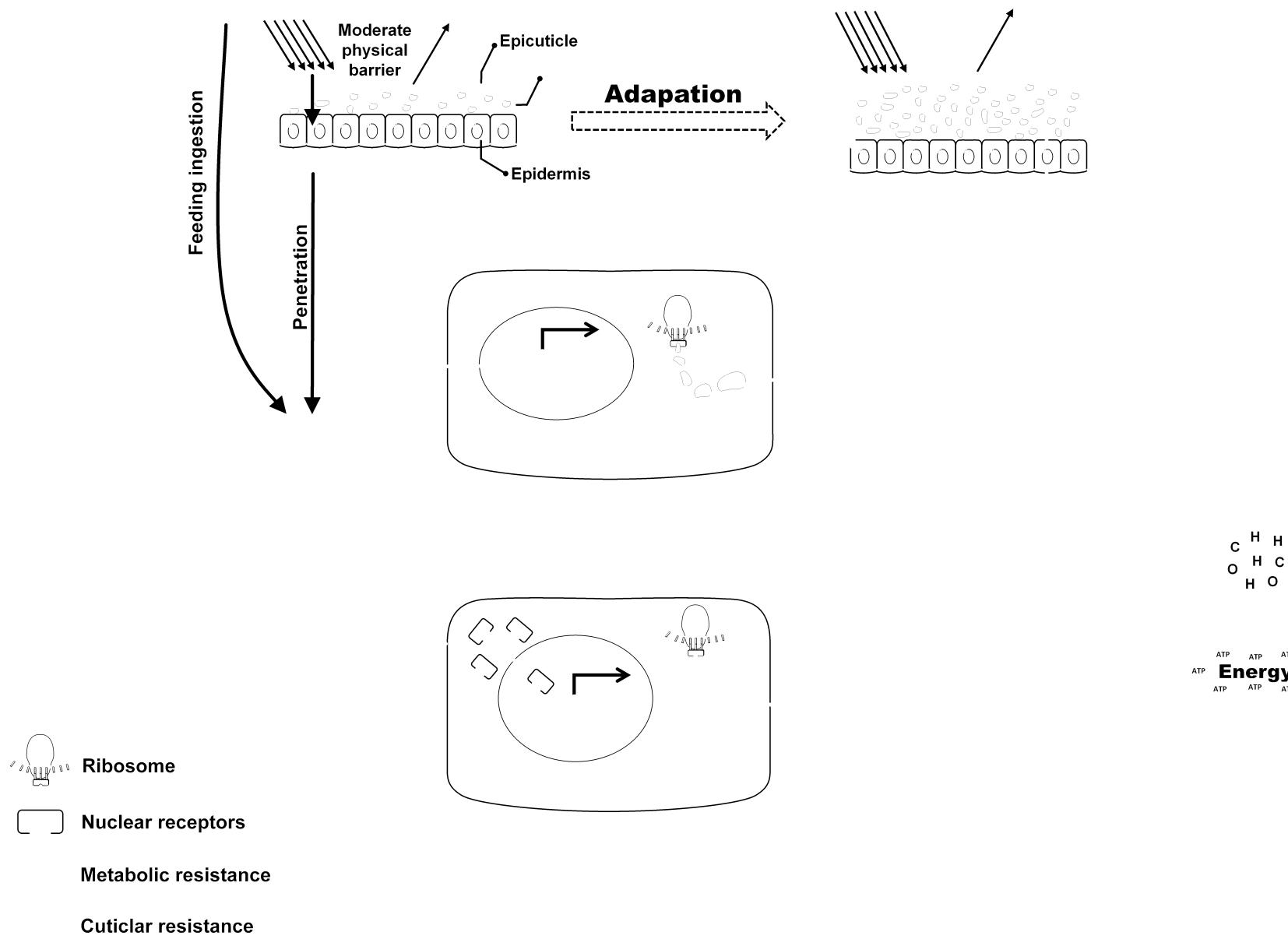

\section{Figure 10}

Overview of the proposed mechanisms underlying resistance to host-specific terpenoid defences in P. tsushimanus larvae. Upon exposure to host terpenoids by means of feeding ingestion and penetration, the larvae deploy cuticular resistance [including $(\mathbb{(})$ activation of the transcriptional capacity of CP genes; $(\mathbb{\nabla})$ massive production of CPs; (『) translocation of CPs; (『) thickening of the procuticle, contributing to an enhanced physical barrier] and metabolic resistance [including $(\mathbb{\nabla})$ capture of terpenoid molecules; $(\mathbb{\nabla})$ activation of the transcriptional capacity of CYP450 genes; ( $₫)$ massive production of CYP450 enzyme;

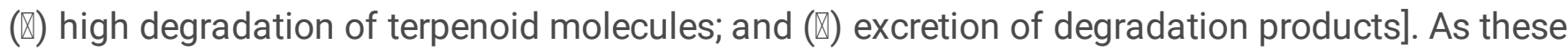
responses occur, certain products may be converted into carbohydrates used for energy, resulting in a stimulatory effect on the larval growth phenotype (i.e., hormesis). 